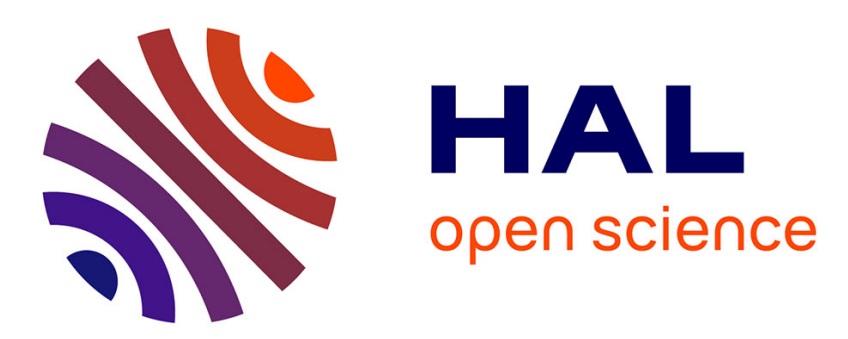

\title{
Parametric solution of the Rayleigh-Benard convection model by using the PGD Application to nanofluids
}

\author{
Saeid Aghighi, Amine Ammar, Christelle Metivier, Francisco Chinesta
}

\section{To cite this version:}

Saeid Aghighi, Amine Ammar, Christelle Metivier, Francisco Chinesta. Parametric solution of the Rayleigh-Benard convection model by using the PGD Application to nanofluids. International Journal of Numerical Methods for Heat and Fluid Flow, 2015, 25 (6), pp.1252-1281. 10.1108/HFF-06-20140196 . hal-01206794

\author{
HAL Id: hal-01206794 \\ https://hal.science/hal-01206794
}

Submitted on 29 Sep 2015

HAL is a multi-disciplinary open access archive for the deposit and dissemination of scientific research documents, whether they are published or not. The documents may come from teaching and research institutions in France or abroad, or from public or private research centers.
L'archive ouverte pluridisciplinaire HAL, est destinée au dépôt et à la diffusion de documents scientifiques de niveau recherche, publiés ou non, émanant des établissements d'enseignement et de recherche français ou étrangers, des laboratoires publics ou privés. 


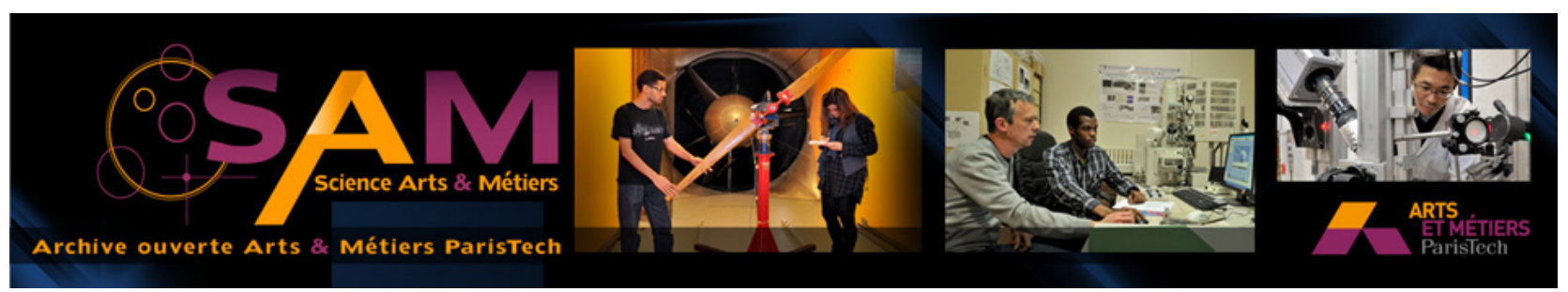

\section{Science Arts \& Métiers (SAM)}

is an open access repository that collects the work of Arts et Métiers ParisTech researchers and makes it freely available over the web where possible.

This is an author-deposited version published in: http://sam.ensam.eu

Handle ID: .http://hdl.handle.net/10985/10242

\section{To cite this version :}

Saeid AGHIGHI, Amine AMMAR, Christelle METIVIER, Francisco CHINESTA - Parametric solution of the Rayleigh-Benard convection model by using the PGD Application to nanofluids International Journal of Numerical Methods for Heat \& Fluid Flow - Vol. 25, n6, p.1252-1281 2015 


\title{
Parametric solution of the Rayleigh-Benard convection model by using the PGD Application to nanofluids
}

\author{
Saeid Aghighi \\ Arts et Metiers, ParisTech, Angers, France and \\ Laboratoire de Rheologie, INPG, Grenoble, France \\ Amine Ammar \\ Arts et Metiers, ParisTech, Angers, France \\ Christelle Metivier \\ LEMTA, Universite de Lorraine, Vandoeuvre-les-Nancy, France, and \\ Francisco Chinesta \\ GEM, UMR CNRS-Centrale Nantes, Nantes, France
}

\section{Abstract}

Purpose - The purpose of this paper is to focus on the advanced solution of the parametric non-linear model related to the Rayleigh-Benard laminar flow involved in the modeling of natural thermal convection. This flow is fully determined by the dimensionless Prandtl and Rayleigh numbers. Thus, if one could precompute (off-line) the model solution for any possible choice of these two parameters the analysis of many possible scenarios could be performed on-line and in real time.

Design/methodology/approach - In this paper both parameters are introduced as model extracoordinates, and then the resulting multidimensional problem solved thanks to the space-parameters separated representation involved in the proper generalized decomposition (PGD) that allows circumventing the curse of dimensionality. Thus the parametric solution will be available fast and easily.

Findings - Such parametric solution could be viewed as a sort of abacus, but despite its inherent interest such calculation is at present unaffordable for nowadays computing availabilities because one must solve too many problems and of course store all the solutions related to each choice of both parameters.

Originality/value - Parametric solution of coupled models by using the PGD. Model reduction of complex coupled flow models. Analysis of Rayleigh-Bernard flows involving nanofluids.

Keywords Nanofluids, Model order reduction, Parametric solutions, PGD, Rayleigh-Benard model, Proper generalized decomposition

Paper type Research paper

\section{Introduction}

Several systems and industrial processes are based on natural convection, justifying the impressive volume of work devoted to its understanding and efficient solution during more than one century. These problems, quite simple in appearance, exhibit many surprises related to their intricate nature and many issues concerning their numerical solution.

In Aghighi et al. (2013) we addressed the transient solution of the Rayleigh-Bénard flow model within a non-incremental framework. For that purpose the different fields involved in the model (the components of the velocity $\mathbf{v}(\mathbf{x}, t)$ and 
temperature $\theta(\mathbf{x}, t)$ fields) were approximated by using a space-time separated representation:

and:

$$
\begin{aligned}
& u(x, y, t) \approx \sum_{i=1}^{i=N} X_{i}^{u}(x, y) \cdot \Theta_{i}^{u}(t) \\
& v(x, y, t) \approx \sum_{i=1}^{i=N} X_{i}^{v}(x, y) \cdot \Theta_{i}^{v}(t) \\
& \theta(x, y, t) \approx \sum_{i=1}^{i=N} X_{i}^{\theta}(x, y) \cdot \Theta_{i}^{\theta}(t)
\end{aligned}
$$

Functions $X_{i}^{u}, X_{i}^{v}$ and $X_{i}^{\theta}$ were calculated by solving a series of two-dimensional (2D) boundary value problems and functions $\Theta_{i}^{u}, \Theta_{i}^{v}$ and $\Theta_{i}^{\theta}$ by solving a series of initial value problems (see Aghighi et al., 2013, for additional details on the solution procedure). Separated representations are involved in the so-called proper generalized decomposition (PGD) methods. The interested reader can refer to some recent reviews on these techniques (Chinesta et al. 2010b, 2011a, b) and the references therein. This non-incremental strategy allowed significant CPU time savings (Aghighi et al., 2013).

The present work constitutes a step forward toward an efficient solution of such coupled non-linear models that govern many systems of industrial interest. The Rayleigh-Bénard flow problem is fully determined by the dimensionless Prandtl $P_{r}$ and Rayleigh $R_{a}$ numbers. Thus, if one could precompute (off-line) the model solution for any possible choice of $P_{r}$ and $R_{a}$, then the analysis of many possible scenarios could be performed on-line and in real time. Moreover, one could explore all the parametric domain and evaluate the impact of any change in the fluid and process conditions. Such parametric solution could be viewed as a sort of computational vademeucm, but despite its inherent interest such calculation is at present unaffordable for nowadays computing availabilities because one must solve too many problems and of course store all the solutions related to each parameters choice.

In this paper we propose an alternative route: $P_{r}$ and $R_{a}$ numbers will be introduced as extra-coordinates, and then the resulting multidimensional problem solved thanks to the space-parameters separated representation involved in the PGD that allows circumventing the curse of dimensionality in the solution of highly multidimensional, parametric and/or coupled models as illustrated in our former works (Ammar et al., 2006, 2007, 2010a; Mokdad et al., 2007; Pruliere et al., 2009; Chinesta et al., 2010a, 2013) and the references therein. The calculation of such a parametric solution, in the non-linear coupled case, by using the PGD constitutes the first original contribution of the present work. The use of the PGD is not the only route for constructing such parametric solution. An alternative route consists in using reduced bases. Interested readers can refer to Maday and Ronquist (2002), Maday et al. (2002), Veroy and Patera (2005), Rozza et al. (2008) and the references therein. Reduced bases were successfully applied for simulating the Rayleigh-Bénard flow model in Herrero et al. (2013) within a framework quite different to the one here considered.

In our former work (Aghighi et al., 2013) we computed a transient solution in a non-incremental manner without considering any parameter as extra-coordinate. 
Thus, the solution was transient but not parametric. In the present work we succeeded to compute a parametric solution of a non-linear flow model where the nonlinearities come from both the inertia term and the thermo-mechanical coupling. It constitutes a first result in that sense. Moreover, the solution procedure is extremely efficient. In fact, we considered 1,000 nodes for discretizing each one of the parametric coordinates, $P_{r}$ and $R_{a}$, that implies a total number of $10^{6}$ flow scenarios. We can imagine that solving one million of non-linear models is not an easy task, however, when using the PGD solver, the computing time is not significantly affected by the mesh involved in the parametric representation because as proved later the problems involved by each parameter is algebraic and 1D. Thus, in few minutes, and by using a standard laptop, the PGD solver calculates a solution that contains the equivalent of one million of 2D non-linear solutions.

In what follows we start by introducing the main ideas of the PGD. The PGD will be then applied in Section 3 for solving the parametric Rayleigh-Bénard flow model. Section 4 presents some numerical results concerning Newtonian fluids and more in particular suspensions involving nano-particles (also known as nanofluids).

It is generally admitted that nanofluids can be considered more as a single-phase fluid than a two-phase fluid. For low particles volume fractions the rheology seems not dependent on the applied shear rate and then the nanofluid can be considered in first approximation as a Newtonian fluid with a viscosity depending on the particles diameter and its volume fraction. Many works make use of this assumption (Massimo, 2011; Abouali and Ahmadi, 2012; Alloui et al., 2011; Cianfrini et al., 2011; Elhajjar et al., 2010). Computational modeling is in any case needed for evaluating the heat transfer efficiency that depends on the flow features, these last influenced by the thermal and rheological properties (Abu-Nada and Chamkha, 2010; Oueslati and Bennacer, 2011; Mahmoudi et al., 2010; Aminossadati and Ghasemi, 2009; Abu-Nada, 2011).

The use of nanofluids (involving a suspension of nano-particles) in industrial applications is experiencing an impressive growth because the significant increase of the thermal conductivity of the base fluid by adding some few percents of nanoparticles. However, some works (Abu-Nada et al., 2010; Massimo, 2011) claimed that in the case of convection dominated heat transfer, as in the case of the one occurring in the Rayleigh-Bénard cavity, the heat transfer seems insensitive to the particles volume fraction, and even sometimes it decreases when increasing the dispersed phase.

For analyzing deeply the question relative to the real effect of nano-particles on convection dominated flows the parametric solution of the flow model seems an appealing route, allowing a fast and accurate exploration of the parametric space. The main conclusion of the analysis addressed in the present work is that the use of nanofluids does not constitute a valuable technological opportunity, but at least such conclusion seems of interest because it is based on a fine analysis of the parametric solution. Independently of the little interest of using nanofluids, the parametric solution that we calculate can be applied to any Newtonian fluid within a Rayleigh-Bénard flow cell, and then such a parametric solution could be of interest in other applications.

\section{The PGD at a glance}

Consider a problem defined in a space of dimension $d$ for the unknown field $u\left(x_{1}, \ldots, x_{d}\right)$. Here, the coordinates $x_{i}$ denote any usual coordinate (scalar or vectorial) related to physical space, time or problem parameters such as material or flow parameters. We seek a solution for $\left(x_{1}, \ldots, x_{d}\right) \in \Omega_{1} \times \ldots \times \Omega_{d}$. 
The PGD yields an approximate solution in the separated form:

$$
u\left(x_{1}, \ldots, x_{d}\right) \approx \sum_{i=1}^{N} F_{i}^{1}\left(x_{1}\right) \cdot \ldots \cdot F_{i}^{d}\left(x_{d}\right) .
$$

The PGD approximation is thus a sum of $N$ functional products involving each a number $d$ of functions $F_{i}^{j}\left(x_{j}\right)$ that are unknown a priori. It is constructed by successive enrichment, whereby each functional product is determined in sequence. At a particular enrichment step $n+1$, the functions $F_{i}^{j}\left(x_{j}\right)$ are known for $i \leqslant n$ from the previous steps, and one must compute the new product involving the $d$ unknown functions $F_{n+1}^{j}\left(x_{j}\right), j=1, \ldots, d$. This is achieved by invoking the weak form of the problem under consideration. The resulting discrete system is non-linear, which implies that iterations are needed at each enrichment step. A low-dimensional problem can thus be defined in $\Omega_{j}$ for each of the $d$ functions $F_{n+1}^{j}\left(x_{j}\right), j=1, \ldots, d$. For additional details on the separated representation constructor the interested reader can refer to Appendix 1.

\subsection{Parametric solution-based vademecum}

In the case of a field depending on the physical space $\mathbf{x} \in \Omega_{x} \subset R^{3}$, the time $\boldsymbol{t} \in \mathcal{I}_{t} \subset \mathcal{R}$ and $Q$ parameters $p^{1}, \ldots, p^{\mathrm{Q}}, p^{j} \in \Omega_{p^{j}}, j=1, \ldots, Q$, the solution is sought under the separated form:

$$
u\left(\mathbf{x}, t, p^{1}, \ldots, p^{Q}\right) \approx \sum_{i=1}^{N} X_{i}(\mathbf{x}) \cdot T_{i}(t) \cdot \prod_{j=1}^{Q} P_{i}^{j}\left(p^{j}\right)
$$

As soon as this solution is available, after solving the multidimensional model within the PGD framework, we can have access to any possible solution. In fact the representation (5) implies an approximation of each function. Thus the space functions $X_{i}(\mathbf{x})$ could be approximated, for example, by using a finite element interpolation that implies knowing the value of each space function $X_{i}(\mathbf{x})$ at the $\mathcal{M}_{x}$ nodes $\mathbf{x}_{k}$ $\left(k=1, \ldots, \mathcal{M}_{x}\right)$ of the mesh used for approximating them, i.e. $X_{i}\left(\mathbf{x}_{k}\right)$. The functions $T_{i}(t)$ depending on time will be expressed from the values of those functions at $\mathcal{M}_{t}$ time instants $t_{l}\left(l=1, \ldots, \mathcal{M}_{\mathrm{t}}\right)$, i.e. $T_{i}\left(t_{l}\right)$. Finally, the functions depending on the different parameters $P_{i}^{j}\left(p^{j}\right)$ will be expressed from the values of those functions at $\mathcal{M}_{p^{j}}$ values of each parameter $p_{r}^{j}\left(r=1, \ldots, \mathcal{M}_{p^{j}}\right)$, i.e. $P_{i}^{j}\left(p_{r}^{j}\right)$. Thus, the solution consists of $N$ vectors of size $\mathcal{M}_{x}$ that contain the discrete representation of functions $X_{i}(\mathbf{x}), N$ vectors of size $\mathcal{M}_{t}$ that contain the discrete representation of functions $T_{i}(t)$ and $N$ vectors of size $\mathcal{M}_{p^{j}}$ that contain the discrete representation of functions $P_{i}^{j}\left(p^{j}\right), j=1, \ldots Q$.

If we imagine that $\mathcal{M}_{x}=\mathcal{M}_{t}=\mathcal{M}_{p^{1}}=\ldots=\mathcal{M}_{p^{Q}}=\mathcal{M}$, then the solution representation involves $N \cdot(Q+2)$ vectors of size $\mathcal{M}$, that is $N \cdot(Q+2) \mathcal{M}$ values instead of the $\mathcal{M}^{2+Q}$ involved by an equivalent mesh. If for a while we imagine $Q=10$, $\mathcal{M}=100$ and $N=10$ the separated representation will involve $10^{4}$ values instead of $10^{20}$ involved in a hypothetical equivalent mesh.

Thus, the PGD solver allowing the construction of the separated representation (5) can be viewed as a solver that constructs on the fly a compressed representation of the model solution. Its connexion with singular value decompositions and its high order counterpart was highlighted in Chinesta et al. (2013) where a PGD-based post-compression was proposed for a posteriori reduction of the number of terms involved by the separated representation. 
Until now, we presented the PGD as en efficient solver, however, it can be also viewed as a model reduction strategy when computing only the most relevant terms of the separated representation. In that case an error is introduced, but because the few number of terms retained, storage and data post-processing can be efficiently performed even on deployed platforms.

As soon as a separated representation is available, it can be viewed as a computational handbook, a metamodel, containing an unimaginable amount of information. For example, if the solution (5) applies for a model involving ten parameters, all them considered as extra-coordinates, i.e. $Q=10$, and ten discrete values are considered for describing each parametric dependency, i.e. $\mathcal{M}_{1}=\cdots=\mathcal{M}_{10}=10$ the solution (5) contains the information associated to $10^{10}$ possible scenarios. Obtaining an equivalent amount of information would require the solution of $10^{10}$ transient $3 \mathrm{D}$ problems. Now, from this extremely rich metamodel, one could obtain the solution for any possible scenario $u\left(\mathbf{x}, t, p^{1}, \ldots, p^{10}\right)$ from the parametric solution (5).

\section{Separated representation of the parametric Rayleigh-Bénard model solution}

We consider the dimensionless form of the Rayleigh-Bénard model (see Appendix 2 for more details concerning the derivation of this model):

$$
\left\{\begin{array}{l}
\nabla \cdot \mathbf{v}=0 \\
\frac{\partial \mathbf{v}}{\partial t}+\mathbf{v} \cdot \nabla \mathbf{v}=-\nabla p+\left(\frac{P_{r}}{R_{a}}\right)^{\frac{1}{2}} \nabla \cdot \mathbf{D}+\theta \mathbf{j} \\
\frac{\partial \theta}{\partial t}+\mathbf{v} \cdot \nabla \theta=\left(P_{r} \cdot R_{a}\right)^{-\frac{1}{2}} \nabla^{2} \theta
\end{array}\right.
$$

where $\mathbf{v}$ is the dimensionless velocity, $p$ is the dimensionless pressure, $\theta$ the dimensionless temperature, $\mathbf{D}$ the dimensionless strain rate tensor (symmetric component of the dimensionless velocity gradient), $P_{r}$ and $R_{a}$ the Prandtl and Rayleigh dimensionless numbers, respectively (theirs expressions are given in the Appendix 2) and $\mathbf{j}$ the unit vector defining the direction along which gravity force applies (the $y$-direction in our case).

The model is defined in the unit square domain $\Omega=(0,1) \times(0,1)$ (related to the square domain of size $H$ depicted in Figure 1) fulfilled by a fluid initially at rest. A dimensionless temperature $\theta(x, y=0)=\theta_{H}=0.5$ is enforced at the bottom boundary

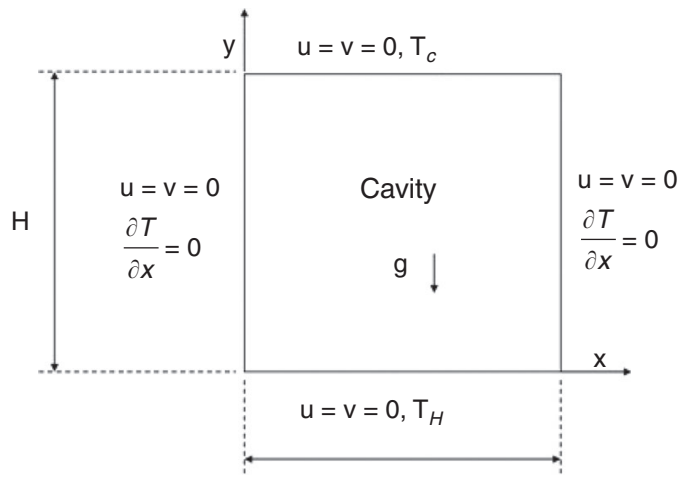


$y=0$ whereas a low dimensionless temperature $\theta(x, y=1)=\theta_{C}=-0.5$ is applied on the upper boundary. The dimensionless temperature $\theta$ is defined by:

$$
\theta=\frac{T-T_{r}}{T_{H}-T_{C}}
$$

where $T_{C}$ and $T_{H}\left(T_{H}>T_{C}\right)$ are the temperatures enforced at the top and bottom cavity boundaries, respectively, and $T_{r}$ is a reference temperature $T_{r}=\left(T_{H}+T_{C}\right) / 2$.

The heat flux is assumed vanishing on the left and right domain boundaries. The initial temperature distribution compatible with a thermal conduction regime in a fluid at rest, evolves linearly in the $y$-direction:

$$
\theta(x, y, t=0)=\theta_{H}-y
$$

Equation (6) defines a mixed formulation involving as unknowns the dimensionless velocity, pressure and temperature fields. When we proceed to the discretization of the weak form related to Equation (6) some stability conditions must be ensured. One of them concerns the so-called LBB condition that restricts the free choice of pressure and velocity approximations. As in Aghighi et al. (2013) in what follows we consider a penalty formulation of the incompressibility constraint. Thus, the mass balance we are considering writes:

$$
\nabla \cdot \mathbf{v}+\frac{1}{\lambda} p=0
$$

with $\lambda$ a large enough constant. This expression implies:

$$
p=-\lambda(\nabla \cdot \mathbf{v})
$$

that substituted in the momentum equation simplifies the model formulation:

$$
\left\{\begin{array}{l}
\frac{\partial \mathbf{v}}{\partial t}+\mathbf{v} \cdot \nabla \mathbf{v}=\lambda \nabla(\nabla \cdot \mathbf{v})+\left(\frac{P_{r}}{R_{a}}\right)^{\frac{1}{2}} \nabla \cdot \mathbf{D}+\theta \mathbf{j} \\
\frac{\partial \theta}{\partial t}+\mathbf{v} \cdot \nabla \theta=\left(P_{r} \cdot R_{a}\right)^{-\frac{1}{2}} \nabla^{2} \theta
\end{array}\right.
$$

which only implies the dimensionless velocity and temperature fields.

The weighted residual form related to Equation (11) reads:

$$
\left\{\begin{array}{l}
\int_{\Omega \times \mathcal{I}} \mathbf{v}^{*} \cdot\left\{\frac{\partial \mathbf{v}}{\partial t}+\mathbf{v} \cdot \nabla \mathbf{v}-\lambda \nabla(\nabla \cdot \mathbf{v})-\left(\frac{P_{r}}{R_{a}}\right)^{\frac{1}{2}} \nabla \cdot \mathbf{D}-\theta \mathbf{j}\right\} d \mathbf{x} d t=0 \\
\int_{\Omega \times \mathcal{I}} \theta^{*} \cdot\left\{\frac{\partial \theta}{\partial t}+\mathbf{v} \cdot \nabla \theta-\left(P_{r} \cdot R_{a}\right)^{-\frac{1}{2}} \nabla^{2} \theta\right\} d \mathbf{x} d t=0
\end{array}\right.
$$

where $\mathcal{I}$ represents the dimensionless time interval.

In the previous model the coefficients $\left(P_{r} / R_{a}\right)^{1 / 2}$ and $\left(P_{r} \cdot R_{a}\right)^{-1 / 2}$ can be viewed as a sort of equivalent viscosity and thermal diffusivity, respectively, that for the sake of notational simplicity could be noted by $\tilde{\eta}$ and $\tilde{a}$, respectively.

Thus the above strong and weak formulations read:

$$
\left\{\begin{array}{l}
\frac{\partial \mathbf{v}}{\partial t}+\mathbf{v} \cdot \nabla \mathbf{v}=\lambda \nabla(\nabla \cdot \mathbf{v})+\tilde{\eta} \nabla \cdot \mathbf{D}+\theta \mathbf{j} \\
\frac{\partial \theta}{\partial t}+\mathbf{v} \cdot \nabla \theta=\tilde{\alpha} \nabla^{2} \theta
\end{array}\right.
$$


and:

$$
\left\{\begin{array}{l}
\int_{\Omega \times \mathcal{I}} \mathbf{v}^{*} \cdot\left\{\frac{\partial \mathbf{v}}{\partial t}+\mathbf{v} \cdot \nabla \mathbf{v}-\lambda \nabla(\nabla \cdot \mathbf{v})-\tilde{\eta} \nabla \cdot \mathbf{D}-\theta \mathbf{j}\right\} d \mathbf{x} d t=0 \\
\int_{\Omega \times \mathcal{I}} \theta^{*} \cdot\left\{\frac{\partial \theta}{\partial t}+\mathbf{v} \cdot \nabla \theta-\tilde{\alpha} \nabla^{2} \theta\right\} d \mathbf{x} d t=0
\end{array}\right.
$$

respectively.

\subsection{Extended parametric weak form}

When both parameters $\tilde{\eta}$ and $\tilde{\alpha}$ are considered as extra-coordinates the weak form must be considered in the extended domain resulting from $\Omega, \mathscr{I}$ and the domains in which both extra-coordinates take theirs values that we will note by $\Xi_{\eta}$ and $\Xi_{\alpha}$, respectively. We define the domain $\Xi=\Xi_{\eta} \times \Xi_{\alpha}$ and the couples $\xi=(\tilde{\eta}, \tilde{\alpha}) \in \Xi$.

The extended weak form writes in this case:

$$
\left\{\begin{array}{l}
\int_{\Omega \times \mathcal{I} \times \Xi} \mathbf{v}^{*} \cdot\left\{\frac{\partial \mathbf{v}}{\partial t}+\mathbf{v} \cdot \nabla \mathbf{v}-\lambda \nabla(\nabla \cdot \mathbf{v})-\tilde{\eta} \nabla \cdot \mathbf{D}-\theta \mathbf{j}\right\} d \mathbf{x} d t d \boldsymbol{\xi}=0 \\
\int_{\Omega \times \mathcal{I} \times \Xi} \theta^{*} \cdot\left\{\frac{\partial \theta}{\partial t}+\mathbf{v} \cdot \nabla \theta-\tilde{\alpha} \nabla^{2} \theta\right\} d \mathbf{x} d t d \xi=0
\end{array}\right.
$$

with the velocity and temperature fields depending now on the physical coordinates $\mathbf{x}$ and $t$ as well as on both parametric coordinates $\tilde{\eta}$ and $\tilde{\alpha}$, that is $\mathbf{v}(\mathbf{x}, t, \boldsymbol{\xi})$ and $\theta(\mathbf{x}, t, \boldsymbol{\xi})$.

In what follows as we are only interested in the long time solution we are considering the steady state solution and then removing all time dependences, fact that results in:

$$
\left\{\begin{array}{l}
\int_{\Omega \times \Xi} \mathbf{\Xi}^{*} \cdot\{\mathbf{v} \cdot \nabla \mathbf{v}-\lambda \nabla(\nabla \cdot \mathbf{v})-\tilde{\eta} \nabla \cdot \mathbf{D}-\theta \mathbf{j}\} d \mathbf{x} d \boldsymbol{\xi}=0 \\
\int_{\Omega \times \Xi} \theta^{*} \cdot\left\{\mathbf{v} \cdot \nabla \theta-\tilde{\alpha} \nabla^{2} \theta\right\} d \mathbf{x} d \boldsymbol{\xi}=0
\end{array}\right.
$$

with $\mathbf{v}(\mathbf{x}, \boldsymbol{\xi})$ and $\theta(\mathbf{x}, \boldsymbol{\xi})$.

\subsection{Separated representation of velocity and temperature fields}

With the components of the velocity field $\mathbf{v}$ denoted by $(u, v)$, the separated representation of the different unknown fields read:

and:

$$
\begin{aligned}
& u(\mathbf{x}, \boldsymbol{\xi}) \approx \sum_{i=1}^{i=N} X_{i}^{u}(\mathbf{x}) \cdot \Theta_{i}^{u}(\boldsymbol{\xi}) \\
& v(\mathbf{x}, \boldsymbol{\xi}) \approx \sum_{i=1}^{i=N} X_{i}^{v}(\mathbf{x}) \cdot \Theta_{i}^{v}(\boldsymbol{\xi})
\end{aligned}
$$

$$
\theta(\mathbf{x}, \boldsymbol{\xi}) \approx \sum_{i=1}^{i=N} X_{i}^{\theta}(\mathbf{x}) \cdot \Theta_{i}^{\theta}(\xi)
$$

with $\mathbf{x}=(x, y)$ and $\boldsymbol{\xi}=(\tilde{\eta}, \tilde{\alpha})$.

In the separated representation of both components of the velocity vector (17) and (18) we considered different functions $\Theta_{i}^{u}(\xi)$ and $\Theta_{i}^{v}(\xi)$ because this choice offers the most compact representation. We could consider identical functions $\Theta_{i}(\xi)$ in the 
separated representation of both velocity components but in that case the number of terms involved in the separated representation would be higher:

Remark 1. As the problem here addressed, the Rayleigh-Bénard flow model, only involves two parameters we decided to consider both parameters grouped when defining the separated representation. Obviously an alternative separated representation could consist of a fully separated representation of the parametric space, that is:

$$
u(\mathbf{x}, \boldsymbol{\xi}) \approx \sum_{i=1}^{i=N^{\prime}} X_{i}^{u}(\mathbf{x}) \cdot \Theta_{i}^{u}(\tilde{\eta}) \cdot \Upsilon_{i}^{u}(\tilde{\alpha})
$$

and similarly for $v$ and $\theta$. This representation involves in general more terms, that is, $N^{\prime}>N$, but allows considering many parameters as extracoordinates without suffering the curse of dimensionality. In general the more are the coordinates considered separately the higher is the number of terms involved in the finite sum. In the limit case in which all the coordinates are considered fully grouped (without any separation) the solution consists in a single term.

Expressions (17) and (18) can be written in the compact separated representation vector form:

$$
\mathbf{v}(\mathbf{x}, \boldsymbol{\xi}) \equiv\left(\begin{array}{l}
u(\mathbf{x}, \boldsymbol{\xi}) \\
v(\mathbf{x}, \boldsymbol{\xi})
\end{array}\right) \approx\left(\begin{array}{l}
\sum_{i=1}^{i=N} X_{i}^{u}(\mathbf{x}) \cdot \Theta_{i}^{u}(\boldsymbol{\xi}) \\
\sum_{i=1}^{i=N} X_{i}^{v}(\mathbf{x}) \cdot \Theta_{i}^{v}(\boldsymbol{\xi})
\end{array}\right)=\sum_{i=1}^{i=N} \mathbf{X}_{i}(\mathbf{x}) \circ \Theta_{i}(\boldsymbol{\xi})
$$

where the symbol "o" denotes the so-called entry-wise, Hadamard or Schur multiplication for vectors. Thus for two generic vectors $\mathbf{a}$ and $\mathbf{b}$, the $i$-component of the entry-wise product $(\mathbf{a} \cdot \mathbf{b})_{i}$ is given by $(\mathbf{a} \circ \mathbf{b})_{i}=\mathbf{a}_{i} \cdot \mathbf{b}_{i}$.

This separated representation is built as described in Appendix 1, by computing a term at each iteration of the PGD constructor. Thus, if we assume at iteration $m$ the solution $\mathbf{v}^{m}$ and $\theta^{m}$ given by:

and:

$$
\mathbf{v}^{m}(\mathbf{x}, \boldsymbol{\xi})=\sum_{i=1}^{i=m} \mathbf{X}_{i}(\mathbf{x}) \circ \Theta_{i}(\boldsymbol{\xi})
$$

$$
\theta^{m}(\mathbf{x}, \boldsymbol{\xi})=\sum_{i=1}^{i=m} X_{i}^{\theta}(\mathbf{x}) \cdot \Theta_{i}^{\theta}(\boldsymbol{\xi})
$$

at iteration $m+1$ we look for the next functional products:

and:

$$
\mathbf{v}^{m+1}(\mathbf{x}, \boldsymbol{\xi})=\sum_{i=1}^{i=m} \mathbf{X}_{i}(\mathbf{x}) \circ \Theta_{i}(\boldsymbol{\xi})+\mathbf{R}(\mathbf{x}) \circ \mathbf{S}(\boldsymbol{\xi})=\mathbf{v}^{m}(\mathbf{x}, \boldsymbol{\xi})+\mathbf{R}(\mathbf{x}) \circ \mathbf{S}(\boldsymbol{\xi})
$$

\subsection{Linearization}

$$
\theta^{m+1}(\mathbf{x}, \boldsymbol{\xi})=\sum_{i=1}^{i=m} X_{i}^{\theta}(\mathbf{x}) \cdot \Theta_{i}^{\theta}(\boldsymbol{\xi})+R^{\theta}(\mathbf{x}) \cdot S^{\theta}(\boldsymbol{\xi})=\theta^{m}(\mathbf{x}, \boldsymbol{\xi})+R^{\theta}(\mathbf{x}) \cdot S^{\theta}(\boldsymbol{\xi})
$$

The previous models are always non-linear because the advective terms $\mathbf{v} \cdot \nabla \mathbf{v}$ and $\mathbf{v} \cdot \nabla \theta$, and coupled. The simplest linearization consists of linearizing at iteration $m+1$ 
the non-linear terms around the solution at the previous iteration $m$. Thus, we can write at iteration $m+1$ :

and:

$$
\mathbf{v}^{m+1} \cdot \nabla \mathbf{v}^{m+1} \approx \mathbf{v}^{m} \cdot \nabla \mathbf{v}^{m+1}
$$

$$
\mathbf{v}^{m+1} \cdot \nabla \theta^{m+1} \approx \mathbf{v}^{m} \cdot \nabla \theta^{m+1}
$$

This simple linearization is not optimal as explained in Ammar et al. (2010b), because the number of terms in the decomposition depends on the convergence rate of the fixed point algorithm and not only on the separability of the approximated solution. An enhanced linearization originally proposed in Ammar et al. (2010b) was considered in Aghighi et al. (2013), where the non-linear terms were evaluated at the previous fixed point solution instead of at the previous enrichment step, however, in this paper we use the above simple linearization, because after computing the parametric solution it will be post-compressed by using the strategy described in Chinesta et al. (2013). Thus, in what follows we are not constrained by the separated representation constructor optimality.

\subsection{Separated representation-based weak form}

By considering the simplest linearization and the approximations at iteration $m$ :

$$
\left\{\begin{array}{l}
\mathbf{v}^{m}(\mathbf{x}, \boldsymbol{\xi}) \approx \sum_{i=1}^{m} \mathbf{X}_{i}(\mathbf{x}) \Theta_{i}(\boldsymbol{\xi}) \\
\theta^{m}(\mathbf{x}, \boldsymbol{\xi}) \approx \sum_{i=1}^{m} X_{i}^{\theta}(\mathbf{x}) \cdot \Theta_{i}^{\theta}(\boldsymbol{\xi})
\end{array}\right.
$$

at iteration $m+1$ the searched solution writes:

$$
\left\{\begin{array}{l}
\mathbf{v}^{m+1}(\mathbf{x}, \boldsymbol{\xi}) \approx \sum_{i=1}^{m} \mathbf{X}_{i} \Theta_{i}+\mathbf{R} \circ \mathbf{S} \\
\theta^{m+1}(\mathbf{x}, \boldsymbol{\xi}) \approx \sum_{i=1}^{m} X_{i}^{\theta} \cdot \Theta_{i}^{\theta}+R^{\theta} \cdot S^{\theta}
\end{array}\right.
$$

where for the sake of clarity we do not specify the dependence of functions $\mathbf{X}_{i}, X_{i}^{\theta}, \mathbf{R}$ and $R^{\theta}$ on $\mathbf{x}$ and $\Theta_{i}, \Theta_{i}^{\theta}, \mathbf{S}$ and $S^{\theta}$ on $\boldsymbol{\xi}$.

The test functions related to (29) write:

$$
\left\{\begin{array}{l}
\mathbf{v}^{*}(\mathbf{x}, \xi)=\mathbf{R}^{*} \circ \mathbf{S}+\mathbf{R} \circ \mathbf{S}^{*} \\
\theta^{*}(\mathbf{x}, \xi)=\left(R^{\theta}\right)^{*} \cdot S^{\theta}+R^{\theta} \cdot\left(S^{\theta}\right)^{*}
\end{array}\right.
$$

By introducing Equations (29) and (30) into the extended weak form (16) it results:

$$
\begin{aligned}
\int_{\boldsymbol{\Omega} \times \Xi}\left(\mathbf{R}^{*} \circ \mathbf{S}+\mathbf{R} \circ \mathbf{S}^{*}\right) \cdot\left\{\left(\sum_{i=1}^{i=m} \mathbf{X}_{i} \circ \boldsymbol{\Theta}_{i}\right) \cdot\left(\sum_{i=1}^{i=m} \nabla\left(\mathbf{X}_{i} \circ \Theta_{i}\right)+\nabla(\mathbf{R} \circ \mathbf{S})\right)\right. \\
-\lambda \sum_{i=1}^{i=m} \nabla\left(\nabla \cdot\left(\mathbf{X}_{i} \circ \boldsymbol{\Theta}_{i}\right)\right)-\lambda \nabla(\nabla \cdot(\mathbf{R} \circ \mathbf{S}))-\tilde{\eta}\left(\sum_{i=1}^{i=m} \nabla \cdot \mathbf{D}_{i}+\nabla \cdot \mathbf{D}\right) \\
\left.-\left(\sum_{i=1}^{i=m} X_{i}^{\theta} \cdot \Theta_{i}^{\theta}+R^{\theta} \cdot S^{\theta}\right) \mathbf{j}\right\} d \mathbf{x} d \boldsymbol{\xi}=0
\end{aligned}
$$


and:

$$
\begin{gathered}
\int_{\Omega \times \Xi}\left(\left(R^{\theta}\right)^{*} \cdot S^{\theta}+R^{\theta} \cdot\left(S^{\theta}\right)^{*}\right) \cdot\left\{\left(\sum_{i=1}^{i=m} \mathbf{X}_{i} \Theta_{i}\right) \cdot\left(\sum_{i=1}^{i=m} \nabla X_{i}^{\theta} \cdot \Theta_{i}^{\theta}+\nabla R^{\theta} \cdot S^{\theta}\right)\right. \\
\left.-\tilde{\alpha}\left(\sum_{i=1}^{i=m} \nabla^{2} X_{i}^{\theta} \cdot \Theta_{i}^{\theta}+\nabla^{2} R^{\theta} \cdot S^{\theta}\right)\right\} d \mathbf{x} d \boldsymbol{\xi}=0
\end{gathered}
$$

where:

$$
\left\{\begin{array}{l}
\mathbf{D}_{i}=\frac{1}{2}\left(\nabla\left(\mathbf{X}_{i} \circ \Theta_{i}\right)+\left(\nabla\left(\mathbf{X}_{i} \circ \Theta_{i}\right)\right)^{T}\right) \\
\mathbf{D}=\frac{1}{2}\left(\nabla(\mathbf{R} \circ \mathbf{S})+(\nabla(\mathbf{R} \circ \mathbf{S}))^{T}\right)
\end{array}\right.
$$

We consider the expression of the different differential operators developed in Aghighi et al. (2013) that allows the explicit application of the differential operators on the spatial functions $\mathbf{X}_{i}, X_{i}^{\theta}, \mathbf{R}$ and $R^{\theta}$.

\subsection{Fixed point alternated direction linearization}

Now for computing functions $\mathbf{R}, R^{\theta}, \mathbf{S}$ and $S^{\theta}$, we proceed as described in Appendix 1, by applying a fixed point alternated direction strategy, that starting from an arbitrary $\mathbf{S}$ and $S^{\theta}$ computes $\mathbf{R}$ and $R^{\theta}$ from Equations (31) and (32). Then from the just calculated couple of functions we can update functions $\mathbf{S}$ and $S^{\theta}$. Both steps are repeated until reaching the fixed point, i.e. until the just computed functions are close enough to the previous ones.

It must be highlighted that when functions $\mathbf{S}$ and $S^{\theta}$ are known, integrals in the parametric domain $\Xi$ in Equations (31) and (32) can be performed leading to a weak form that only involves the fields $\mathbf{R}, R^{\theta}$ and theirs derivatives. Obviously, because the original problem involves second order space derivatives of the velocity components and the temperature field, the resulting weak form will involve second order space derivatives on both, the components of $\mathbf{R}$ and $R^{\theta}$. Obviously, integration by parts can by then performed in order to reduce the derivatives order and proceed to the discretization of the resulting weak form by using standard continuous finite element interpolations for example, but other choices exist. A strong form could be derived, as shown in Appendix 1, and then solved by using any collocation technique applied on it.

On the other hand, when functions $\mathbf{R}$ and $R^{\theta}$ are assumed known, space integrals in the space domain $\Omega$ in Equations (31) and (32) can be performed, leading to a weak form that only concerns parametric functions $\mathbf{S}, S^{\theta}$. As the original problem does not involve derivatives with respect to the model parameters $\tilde{\eta}$ and $\tilde{a}$ the resulting strong form defines a simple algebraic problem.

\section{Numerical results}

In what follows we are applying the technique just described to the solution of the Rayleigh-Bénard problem for both Newtonian fluids and suspensions containing nano-particles.

For approximating functions depending on the physical space $\mathbf{x}=(x, y)$ : $\mathbf{X}_{i}, \mathbf{R}, X_{i}^{\theta}$ and $R^{\theta}$ we use standard eight-nodes quadrilateral $\mathrm{C}^{0}$ finite elements. The considered computational meshes of the square cavity involve 1,160 and 2,296 nodes. The finer 
mesh was used to conclude on the convergence of the results computed on the coarser one.

The parametric domain $\Xi=\Xi_{\eta} \times \Xi_{\alpha}$ was defined in order to cover the domain of variation of both the Prandtl and the Rayleigh numbers, that we considered taking values in the intervals $P_{r} \in[0.7,7]$ and $R_{a} \in\left[10^{3}, 10^{5}\right]$. This choice implies $\tilde{\eta} \in \Xi_{\eta}=$ $[0.0026,0.083]$ and $\tilde{\alpha} \in \Xi_{\alpha}=[0.001,0.04]$. The mesh considered in the parametric domain $\Xi=\Xi_{\eta} \times \Xi_{\alpha}$ involves $30 \times 30$ nodes. Convergence is assumed reached when the L2 norm of the error related to the equation residual attains the value $\varepsilon=10^{-6}$. In general 20 terms in the finite sums suffice for solving very accurately both problems. The comparison with the solutions obtained by using the finite element method for some choices of the parameters revealed errors lower that 0.1 percent.

In order to quantify the heat transfer efficiency we define the mean Nusselt number $N_{u}$ from:

$$
N_{u}(\boldsymbol{\xi})=\left.\int_{0}^{1} \frac{\partial \theta(\mathbf{x}, \boldsymbol{\xi})}{\partial y}\right|_{y=0} d x
$$

\subsection{Newtonian fluid}

After solving Equations (31) and (32) in $\Omega \times \Xi$ both parametric solutions $\mathbf{v}(\mathbf{x}, \boldsymbol{\xi})$ and $\theta(\mathbf{x}, \boldsymbol{\xi})$ are available and can be particularized easily and in real time at each position $\mathbf{x} \in \Omega$ and for each couple $\boldsymbol{\xi} \in \boldsymbol{\Xi}$, with $\boldsymbol{\xi}=(\tilde{\eta}, \tilde{\alpha})$ that can be obtained from the values of the dimensionless Prandtl and Rayleigh numbers from:

$$
\left\{\begin{array}{l}
\tilde{\eta}=\left(\frac{P_{r}}{R_{a}}\right)^{\frac{1}{2}} \\
\tilde{\alpha}=\left(P_{r} \cdot R_{a}\right)^{-\frac{1}{2}}
\end{array}\right.
$$

Figure 2 compares the evolution of the maximum value of the x-component of the velocity vector with the Prandtl number for three different values of the Rayleigh number (on the left) and its evolution with the Rayleigh number for three values of the Prandtl number (on the right).

The thermal efficiency is quantified from the evolution of the mean Nusselt number with both, the Prandt and the Rayleigh numbers. Both evolutions are compared in Figure 3.

It is important noticing that all the previous representations only required a single solution of the parametric model in order to compute the general parametric solution
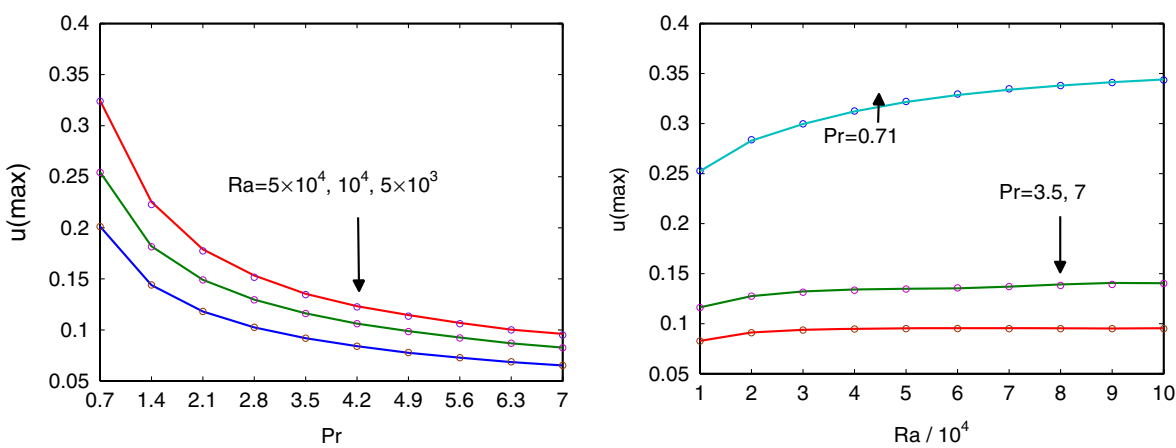
that contains the model solution for any choice of the model parameters in $\Xi$. Thus, the analysis, optimization or the inverse analysis can be performed efficiently because the calculation of the velocity and temperature fields only involves a simple particularizetion of the general parametric solution.

\subsection{Nanofluid}

In this section we are focussing on suspensions involving nano-particles. In particular we are considering the $\mathrm{Al}_{2} \mathrm{O}_{3}$-water suspension for which there is an extensive literature covering its rheological and thermal properties.

In order to use the parametric solution just obtained $\mathbf{v}(\mathbf{x}, \boldsymbol{\xi})$ and $\theta(\mathbf{x}, \boldsymbol{\xi})$ we need deriving the dependence of the model parameters $P_{r}$ and $R_{a}$ on the suspension features (size of the particles and solid phase concentration).

As in the present case we can differentiate two phases, the solid and the suspending fluid, we introduce the indexes $\bullet_{f}$ and $\bullet_{s}$, respectively. When no index is specified the property is related to the suspension itself, that is, to the nanofluid.

The dimensionless Prandtl $\left(P_{r}\right)$ and Rayleigh $\left(R_{a}\right)$ numbers, the first defined from the ratio of momentum diffusivity to thermal diffusivity, and the second one related with buoyancy-driven flows write:

$$
P_{r}=\frac{2 \eta C_{p}}{k}
$$

and:

$$
R_{a}=\frac{g \beta H^{3} \Delta T \rho}{2 \eta \alpha}
$$

respectively, where $\nu$ is the kinematic viscosity.

For obtaining both numbers $\left(P_{r}\right.$ and $\left.R_{a}\right)$ for the suspension we need to derive the expressions given its specific heat, the heat expansion, the viscosity and thermal diffusivity.

For that purpose we start considering a mixture rule for the density:

$$
\rho=\phi \rho_{s}+(1-\phi) \rho_{f}
$$

where $\phi$ is the nano-particles volume fraction.
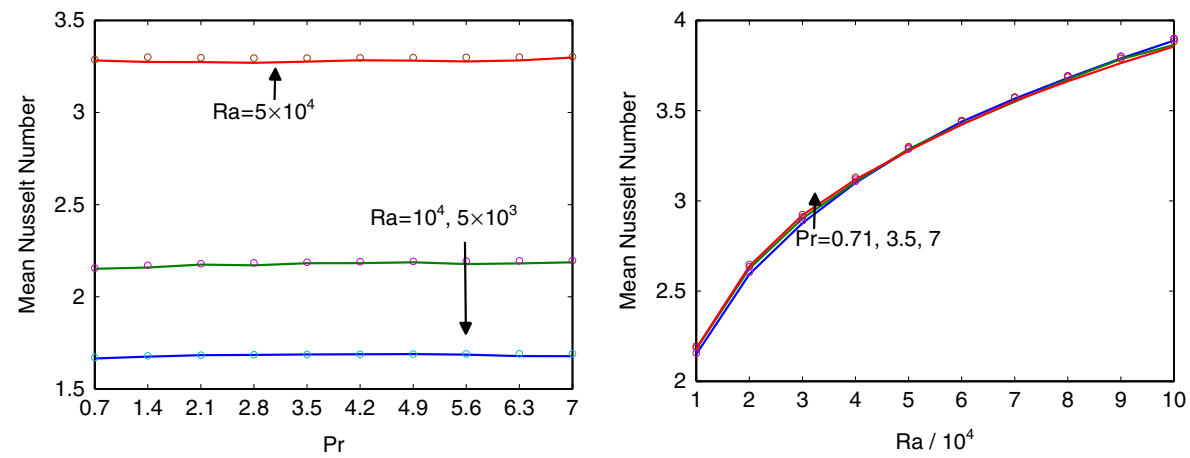
In a similar way we can define for the volumetric heat capacity $\rho \cdot C_{p}$ :

$$
\rho C_{p}=\phi\left(\rho C_{p}\right)_{s}+(1-\phi)\left(\rho C_{p}\right)_{f}
$$

that allows writing:

$$
C_{p}=\frac{\phi\left(\rho C_{p}\right)_{s}+(1-\phi)\left(\rho C_{p}\right)_{f}}{\rho}
$$

with the suspension density $\rho$ given in Equation (38).

By repeating the same reasoning for the thermal expansion we obtain:

$$
\beta=\frac{\phi(\rho \beta)_{s}+(1-\phi)(\rho \beta)_{f}}{\rho}
$$

The thermal conductivity and the viscosity do not accept a mixture rule. In Massimo (2011), authors proposed the following expression for the effective thermal conductivity of nanofluids:

$$
\frac{k}{k_{f}}=1.44 R_{e}^{0.4}\left(P_{r}\right)_{f}^{0.66}\left(\frac{T}{T_{f r}}\right)^{10}\left(\frac{k_{s}}{k_{f}}\right)^{0.03} \phi^{0.66}
$$

where $T_{f r}$ is the freezing temperature of the suspending fluid and $R_{e}$ the Reynolds number defined from:

$$
R_{e}=\frac{2 \rho_{f} K_{b} T}{2 \eta_{f}^{2} d_{p}}
$$

where $K_{b}$ is the Boltzmann's constant and $d_{p}$ the particles diameter.

In the same publication (Massimo, 2011) authors propose an expression for the suspension viscosity:

$$
\frac{\eta}{\eta_{f}}=\frac{1}{1-34.87\left(\frac{d_{p}}{d_{f}}\right)^{-0.3} \phi^{1.03}}
$$

where $d_{f}$ is the equivalent diameter of a fluid molecule calculated from:

$$
d_{f}=0.1\left(\frac{6 M_{m}}{N_{a} \pi \rho_{f 0}}\right)^{\frac{1}{3}}
$$

where $M_{m}$ is the fluid molecular weight, $N_{a}$ is the Avogadro's number and $\rho_{f 0}$ is the fluid density at $293 \mathrm{~K}$.

Thus, as soon as the fluid and the particles properties are known, the ones of the suspension can be obtained and from these both the Prandtl and Rayleigh numbers. In the present model the thermal properties and the viscosity of the suspending fluid are modified by the inclusion of nano-particles, however, its rehological behaviors 
remains Newtonian (the viscosity does not evolve with the shear rate). More complex rheologies (rheothining) where considered in our former work (Aghighi et al., in press) and could be also considered in a parametric framework.

In the numerical results presented below different temperatures $(T=294 \mathrm{~K}$ and $T=324 \mathrm{~K})$, diameters of the nano-particles $\left(d_{p}=25 \mathrm{~nm}\right.$ and $\left.d_{p}=100 \mathrm{~nm}\right)$ and different nano-particles volume fractions (25 values uniformly distributed in the interval $[0,6]$ percent) were considered.

The Prandtl and Rayleigh numbers previously defined can be rewritten as:

$$
P_{r}=\frac{2 \eta C_{p}}{k}=\frac{P_{r}}{\left(P_{r}\right)_{f}}\left(P_{r}\right)_{f}
$$

and:

$$
R_{a}=\frac{g \beta H^{3} \Delta T \rho}{2 \eta \alpha}=\frac{R_{a}}{(R a)_{f}}\left(R_{a}\right)_{f}
$$

Figures 4 and 5 depict the evolution of the mean Nusselt number with the particles volume fraction $\phi$ for different $\left(R_{a}\right)_{f}$, temperature $T$ and particles diameter $d_{p}: T=294 \mathrm{~K}$ and $d_{p}=25 \mathrm{~nm}$ (Figure 4-left); $T=294 \mathrm{~K}$ and $d_{p}=100 \mathrm{~nm}$ (Figure 4-right); $T=324 \mathrm{~K}$ and $d_{p}=25 \mathrm{~nm}$ (Figure 5-left) and $T=324 \mathrm{~K}$ and $d_{p}=100 \mathrm{~nm}$ (Figure 5-right). As expected the heat transfer efficiency increases with the fluid Rayleigh number $\left(R_{a}\right)_{f}$ because the main transfer mechanism is due to convection. Decreasing viscosity enhances the convective effects. Thus, even if the introduction of nano-particles increases the thermal conductivity, its effect can be suppressed by the viscosity increase. Some profiles show a maximum for a given concentration that represents the optimal compromise between the antagonist effects of increasing thermal conductivity and viscosity.

Figures 6-13 depict the steady velocity field and the temperature profiles for different suspensions characterized by $\phi, T, d_{p}$ and $\left(R_{a}\right)_{f}$. As expected convection increases when increasing the Rayleigh number and decreases slightly by increasing the nano-particles volume fraction.

\section{Conclusions}

In this work we considered successfully the parametric solution of non-linear coupled models related to the steady state Rayeigh-Bénard flow model of both Newtonian and nanofluids, by using the PGD. PGD proceeds by building-up a space-parameters separated representation of the different unknown fields, in our case the two components of the velocity field and the temperature. About 20 terms were needed to represent these fields, number that implied the necessity of solving few tens of $2 \mathrm{D}$ problems for calculating the solution of the flow model for any choice of the dimensionless Prandtl and Rayleigh numbers that fully define the flow, instead the thousands required when using standard discretizations procedures that requires a model solution for each couple $\left(P_{r}, R_{a}\right)$. Thus, impressive computing time savings were obtained making possible the fully exploration of the parametric space, that is too expensive when using standard solution procedures. 

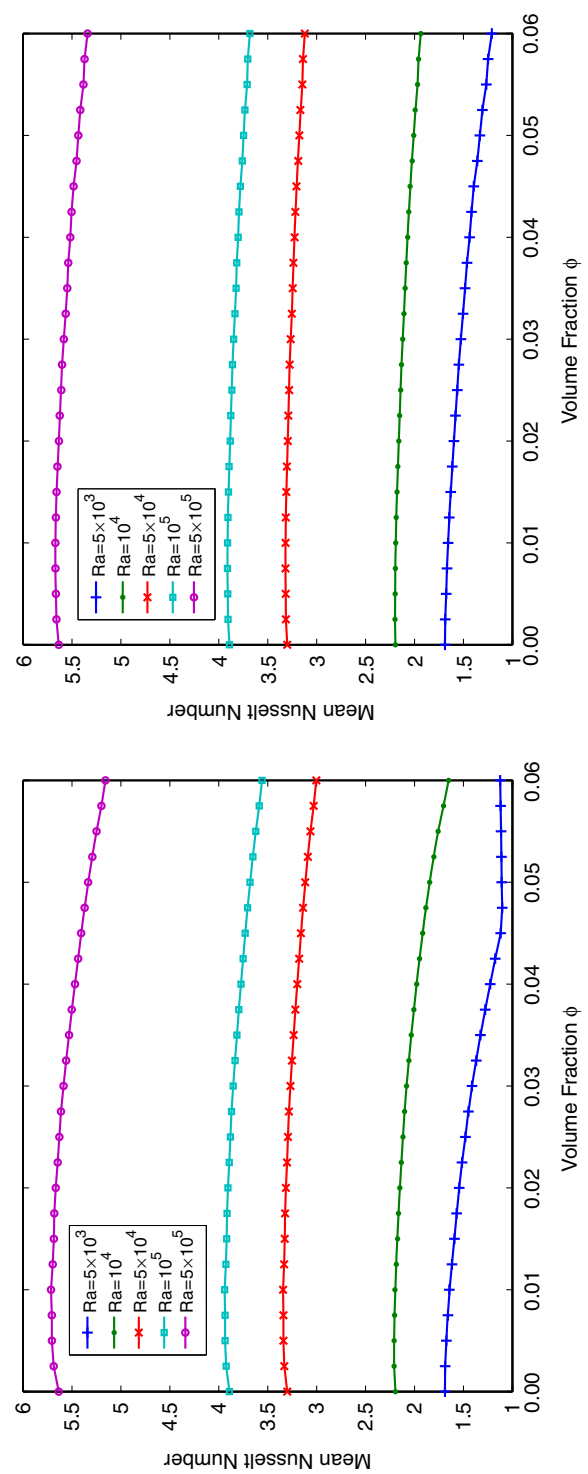

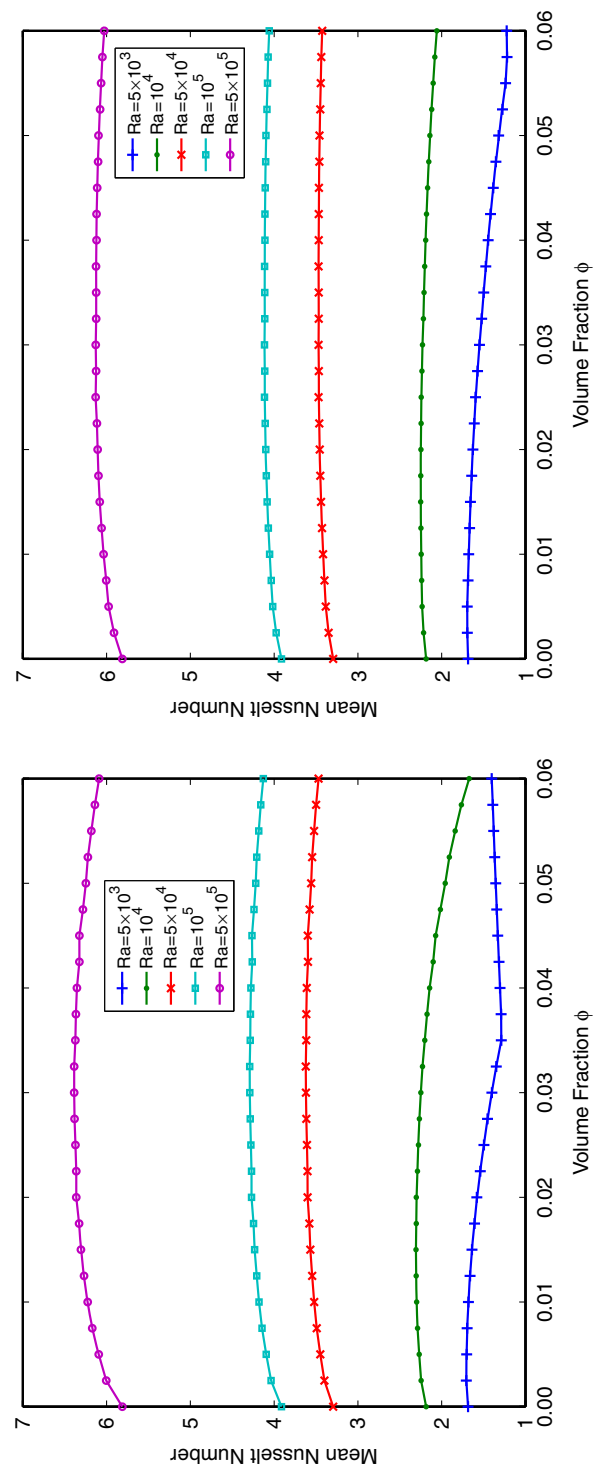

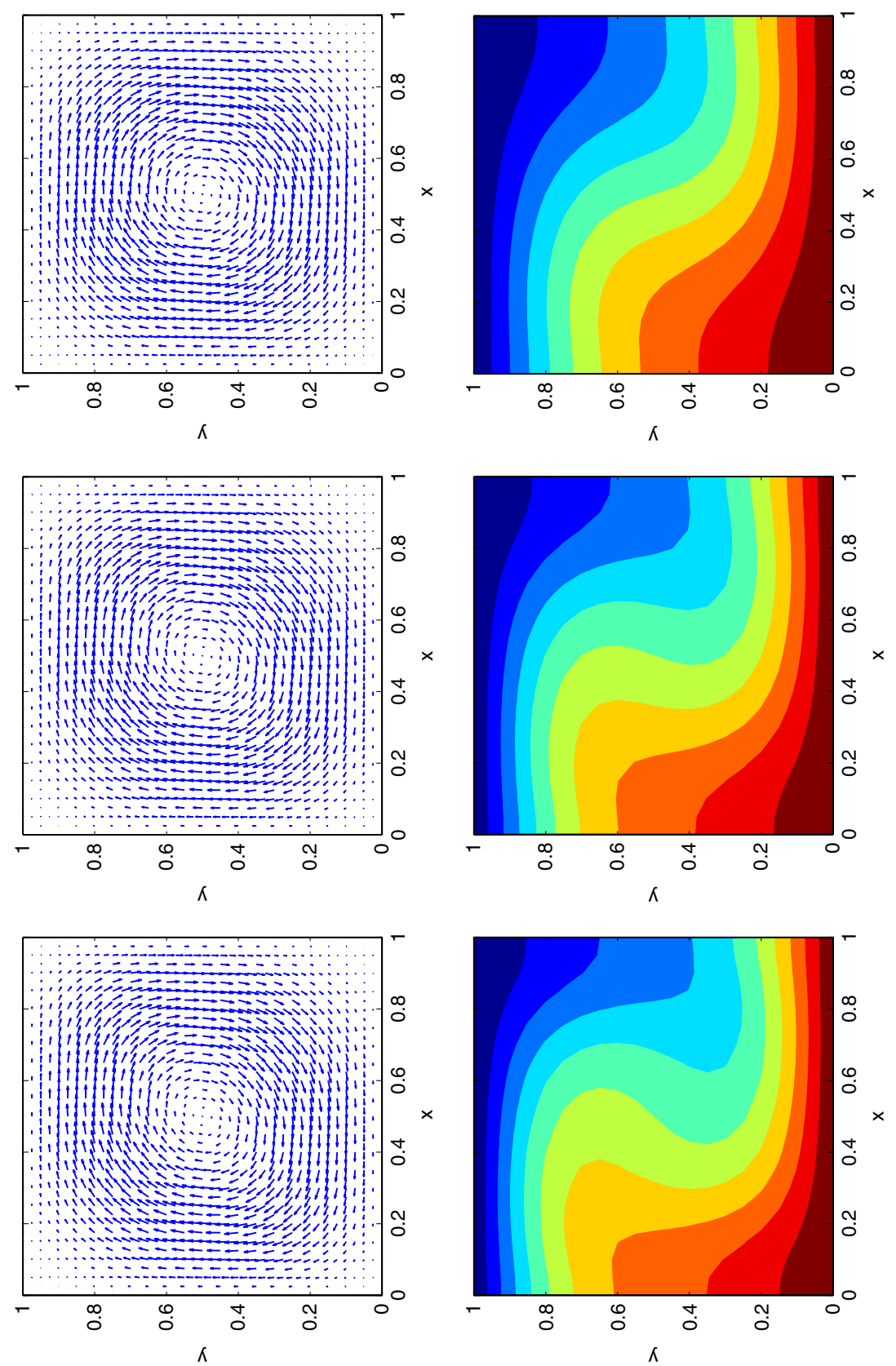

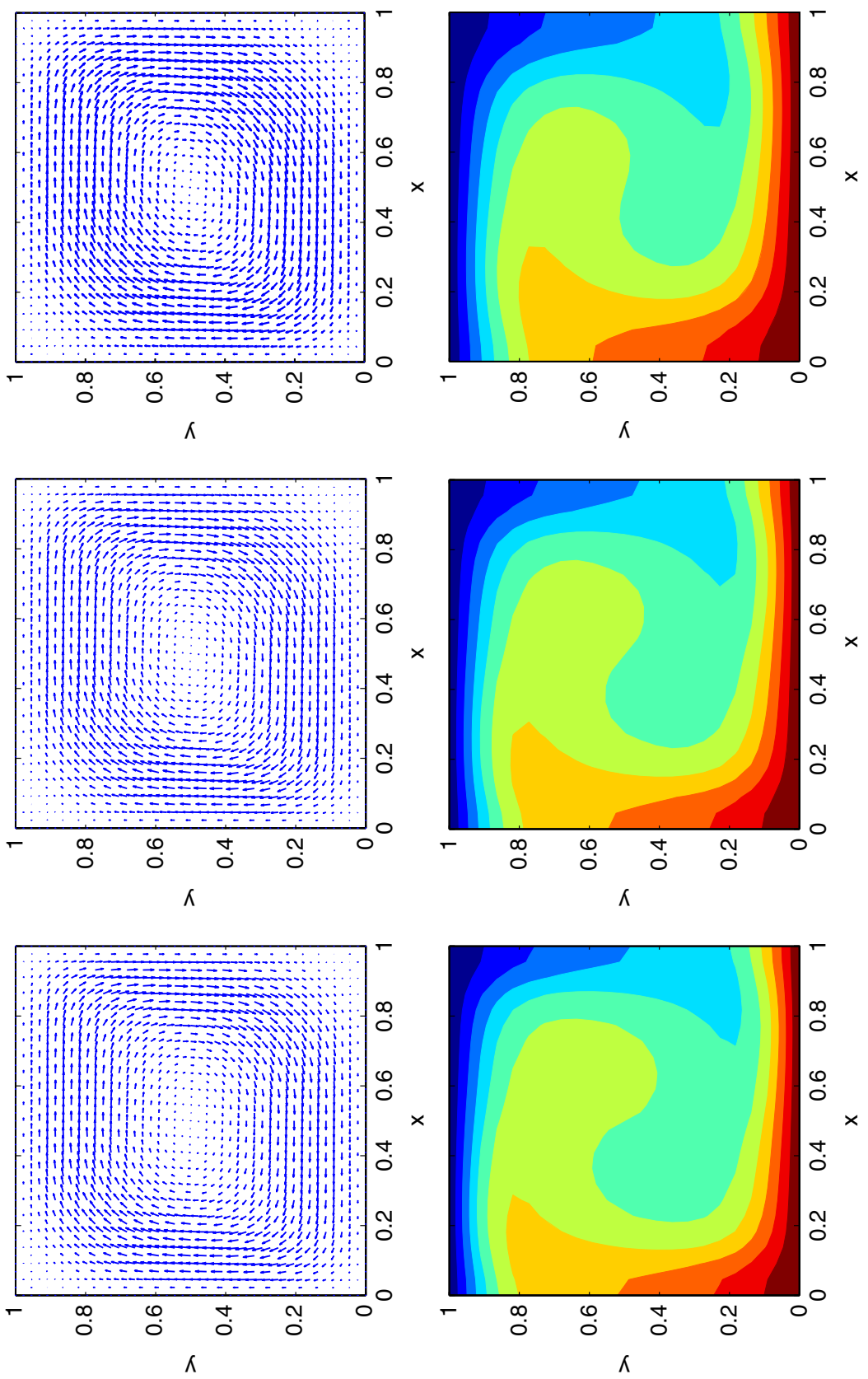

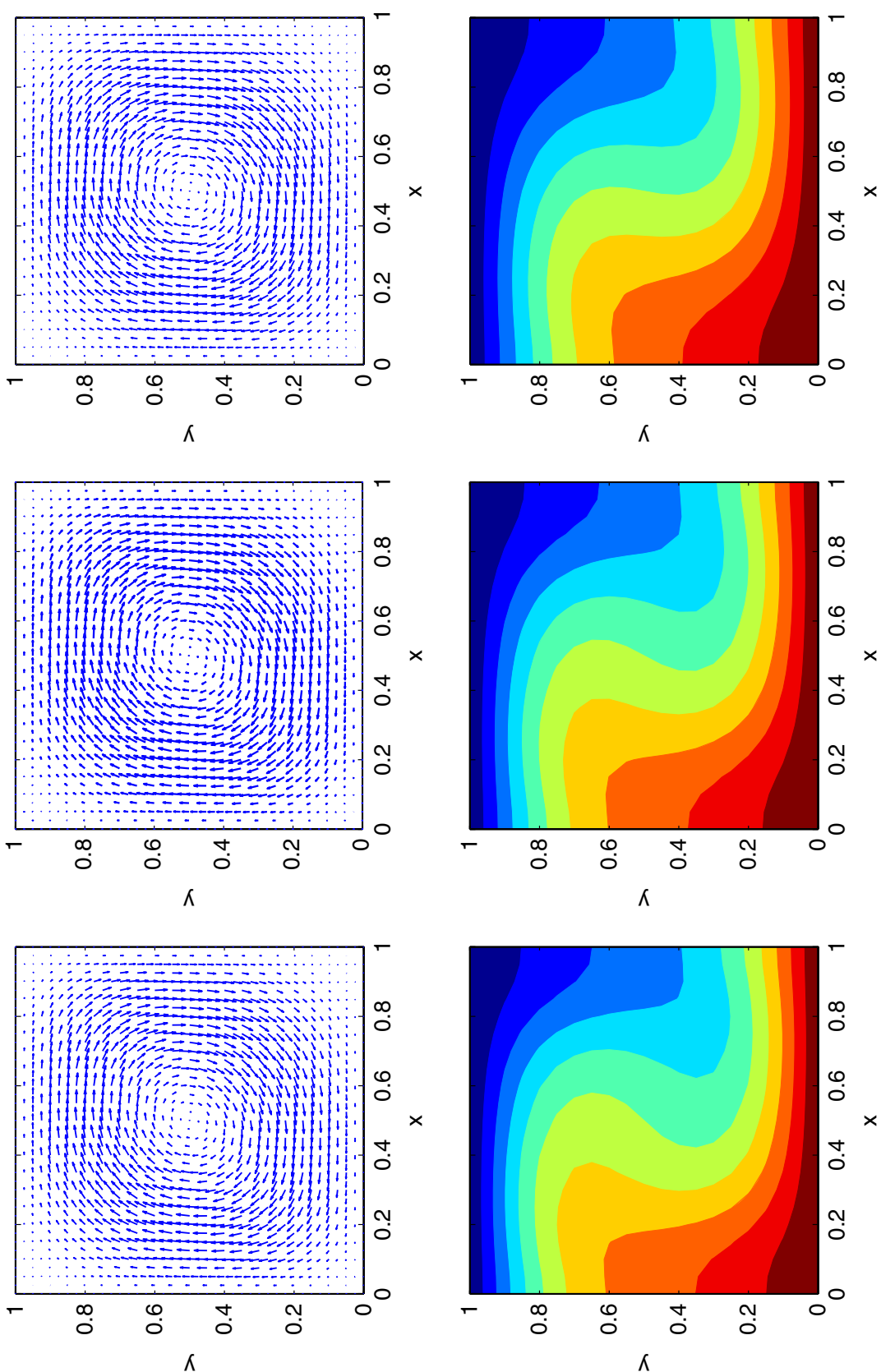

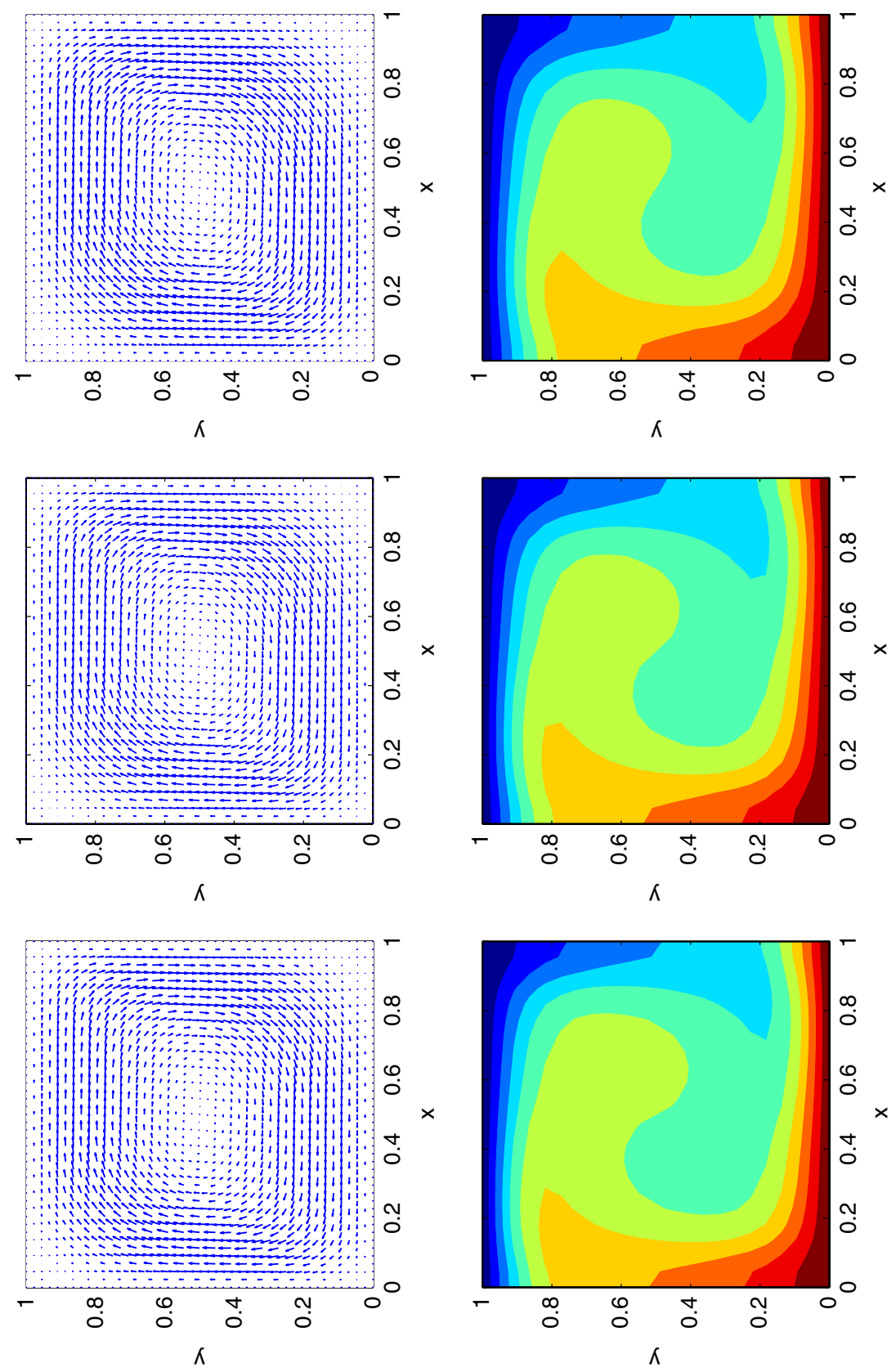

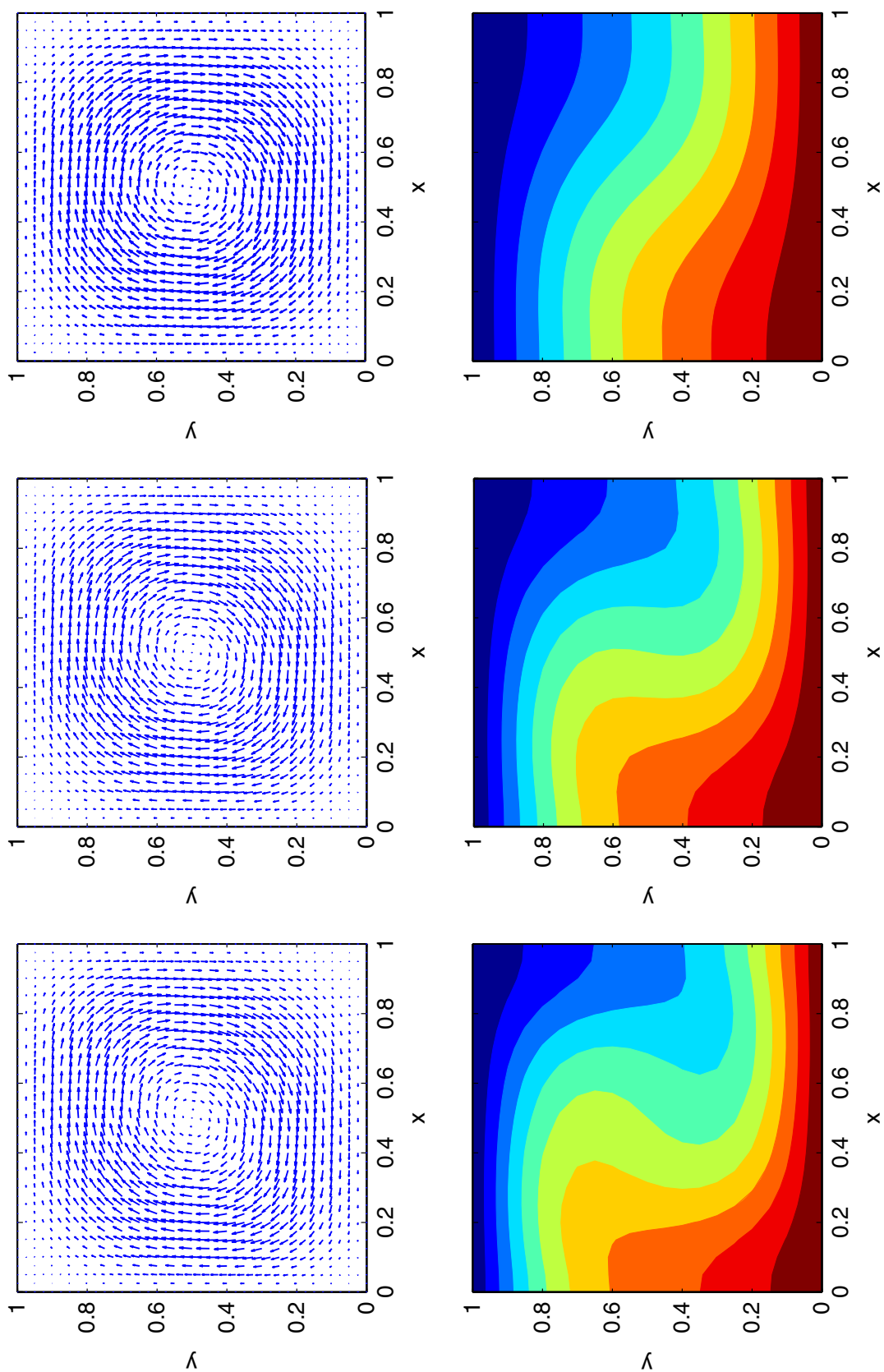

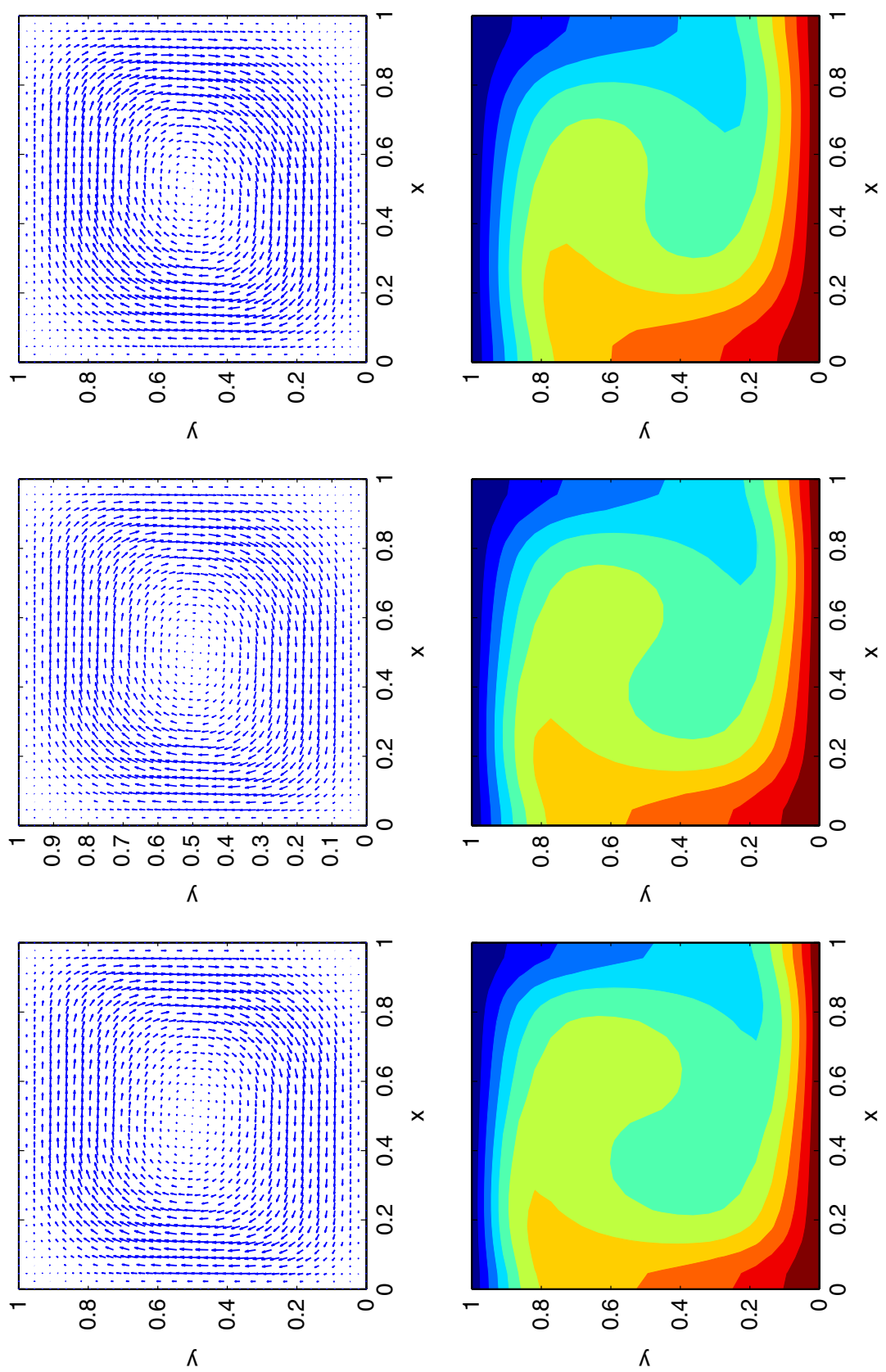

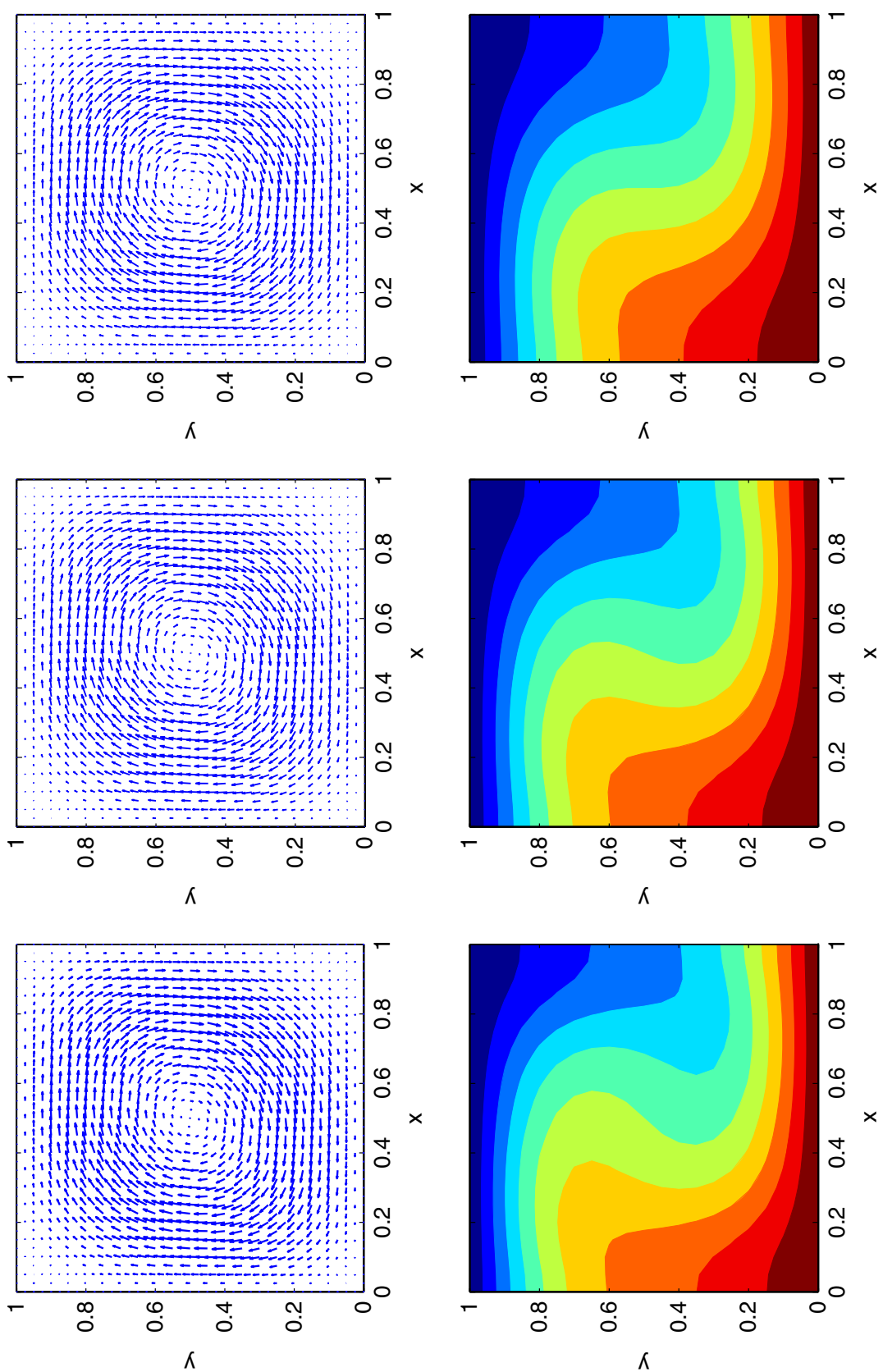

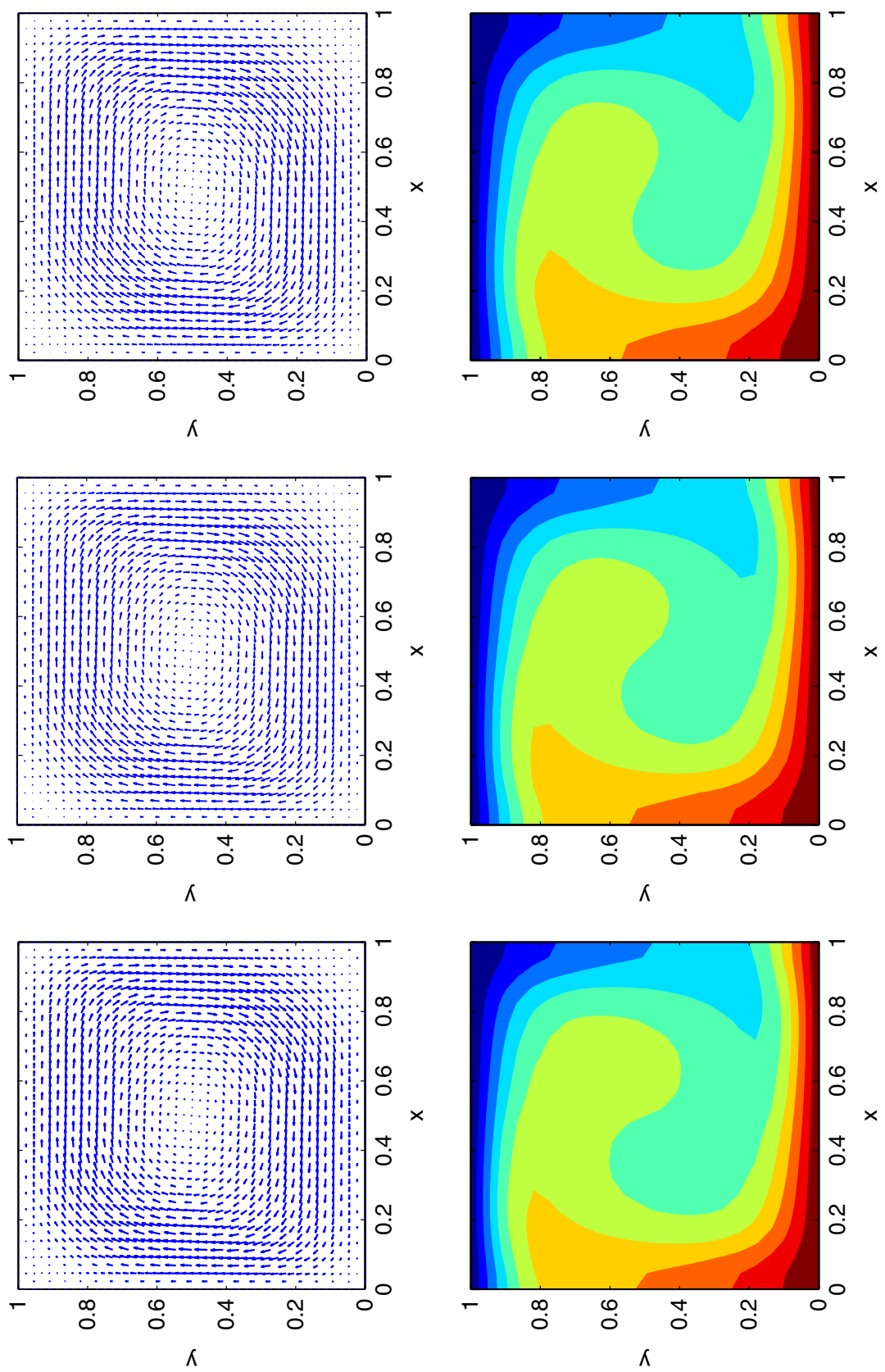


\section{References}

Abouali, O. and Ahmadi, G. (2012), "Computer simulations of natural convection of single phase nanofluids in simple enclosures: a critical review", Applied Thermal Engineering, Vol. 36, pp. 1-13.

Abu-Nada, E. (2011), "Rayleigh-Bénard convection in nanofluids: effect of temperature dependent properties", International Journal of Thermal Sciences, Vol. 50 No. 8, pp. 1720-1730.

Abu-Nada, E. and Chamkha, A.J. (2010), "Effect of nanofluid variable properties on natural convection in enclosures filled with a CuO-EG-Water nanofluid", International Iournal of Thermal Sciences, Vol. 49 No. 12, pp. 2339-2352.

Abu-Nada, E., Masoud, Z., Oztop, H.F. and Campo, A. (2010), "Effect of nanofluid variable properties on natural convection in enclosures", Int. I. Therm. Sci. Vol. 49 No. 3, pp. 479-491.

Aghighi, M.S., Ammar, A., Metivier, C., Normandin, M. and Chinesta, F. (2013), "Non-incremental transient solution of the Rayleigh-Bénard convection model by using the PGD", L. Non-Newtonian Fluid Mech., Vol. 200, pp. 65-78, doi:10.1016/j.jnnfm.2012.11.007.

Alloui, Z., Vasseur, P. and Reggio, M. (2011), "Natural convection of nanofluids in a shallow cavity heated from below", Ó International Iournal of Thermal Sciences, Vol. 50 No. 3, pp. 385-393.

Aminossadati, S.M. and Ghasemi, B. (2009), "Natural convection cooling of a localised heat source at the bottom of a nanofluid-filled enclosure", Eur. I. Mech. B-Fhids, Vol. 28 No. 5, pp. 630-640.

Ammar, A., Normandin, M. and Chinesta, F. (2010a), "Solving parametric complex fluids models in rheometric flows", Journal of Non-Newtonian Fluid Mechanics, Vol. 165 Nos 23-24, pp. 1588-1601.

Ammar, A., Normandin, M., Daim, F., Gonzalez, D., Cueto, E. and Chinesta, F. (2010b), "Nonincremental strategies based on separated representations: applications in computational rheology", Communications in Mathematical Sciences, Vol. 8 No. 3, pp. 671-695.

Ammar, A., Mokdad, B., Chinesta, F. and Keunings, R. (2006), "A new family of solvers for some classes of multidimensional partial differential equations encountered in kinetic theory modeling of complex fluids", L. Non-Newtonian Fluid Mech., Vol. 139 No. 3, pp. 153-176.

Ammar, A., Mokdad, B., Chinesta, F. and Keunings, R. (2007), "A new family of solvers for some classes of multidimensional partial differential equations encountered in kinetic theory modeling of complex fluids. Part II: transient simulation using space-time separated representations", L. Non-Newtonian Fluid Mech., Vol. 144 Nos 2-3, pp. 98-121.

Chinesta, F., Ammar, A. and Cueto, E. (2010a), "Proper generalized decomposition of multiscale models", International Iournal for Numerical Methods in Engineering, Vol. 83 Nos 8-9, pp. 1114-1132.

Chinesta, F., Ammar, A. and Cueto, E. (2010b), "Recent advances and new challenges in the use of the proper generalized decomposition for solving multidimensional models", Archives of Computational Methods in Engineering, Vol. 17 No. 4, pp. 327-350.

Chinesta, F., Ladeveze, P. and Cueto, E. (2011a), "A short review in model order reduction based on proper generalized decomposition", Archives of Computational Methods in Engineering, Vol. 18 No. 4, pp. 395-404.

Chinesta, F., Ammar, A., Leygue, A. and Keunings, R. (2011b), "An overview of the proper generalized decomposition with applications in computational rheology", Iournal of Non Newtonian Fluid Mechanics, Vol. 166 No. 11, pp. 578-592.

Chinesta, F., Leygue, A., Bordeu, F., Aguado, J.V., Cueto, E., Gonzalez, D., Alfaro, I., Ammar, A. and Huerta, A. (2013), "PGD-based computational vademecum for efficient design, optimization and control", Arch Comput Methods Eng., Vol. 20 No. 1, pp. 31-59.

Cianfrini, M., Corcione, M. and Quintino, A. (2011), "Natural convection heat transfer of nanofluids in annular spaces between horizontal concentric cylinders", Applied Thermal Engineering, Vol. 31 Nos 17-18, pp. 4055-4063. 
Elhajjar, B., Bachir, G., Mojtabi, A., Fakih, C. and Charrier-Mojtabi, M.C. (2010), "Modeling of Rayleigh-Bénard natural convection heat transfer in nanofluids", Comptes Rendus Mécanique, Vol. 338 No. 6, pp. 350-354.

Gonzalez, D., Ammar, A., Chinesta, F. and Cueto, E. (2010), "Recent advances in the use of separated representations", International Journal for Numerical Methods in Engineering, Vol. 81 No. 5, pp. 637-659.

Herrero, H., Maday, Y. and Pla, F. (2013), "RB (Reduced basis) for RB (Rayleigh-Bénard)”, Comput. Methods Appl. Mech. Engrg. Vols 261-262, pp. 132-141.

Maday, Y. and Ronquist, E.M. (2002), "A reduced-basis element method”, C. R. Acad. Sci. Paris. Ser. I, Vol. 335, pp. 195-200.

Maday, Y., Patera, A.T. and Turinici, G. (2002), "A priori convergence theory for reduced-basis approximations of single-parametric elliptic partial differential equations", Lournal of Scientific Combuting, Vol. 17 No. 1-4, pp. 437-446.

Mahmoudi, A.H., Shahi, M., Raouf, A.H. and Ghasemian, A. (2010), "Numerical study of natural convection cooling of horizontal heat source mounted in a square cavity filled with nanofluid", International Communications in Heat and Mass Transfer, Vol. 37 No. 1, pp. 1135-1141.

Massimo, C. (2011), "Rayleigh-Bénard convection heat transfer in nanoparticle suspensions", International Iournal of Heat and Fluid Flow, Vol. 32 No. 1, pp. 65-77.

Mokdad, B., Pruliere, E., Ammar, A. and Chinesta, F. (2007), "On the simulation of kinetic theory models of complex fluids using the Fokker-Planck approach", Applied Rheology, Vol. 17 No. 2, pp. 26494-1-26494-14.

Oueslati, F.S. and Bennacer, R. (2011), "Heterogeneous nanofluids: natural convection heat transfer enhancement”, Nanoscale Research Letters, Vol. 6 No. 222, pp. 1-11.

Pruliere, E., Ammar, A., El Kissi, N. and Chinesta, F. (2009), "Recirculating flows involving short fiber suspensions: numerical difficulties and efficient advanced micro-macro solvers", Archives of Computational Methods in Engineering. State of the Art Reviews, Vol. 16 No. 1, pp. 1-30.

Rozza, G., Huynh, D.B.P. and Patera, A.T. (2008), "Reduced basis approximation and a posteriori error estimation for affinely parametrized elliptic coercive partial differential equations application to transport and continuum mechanics", Archives of Computational Methods in Engineering, Vol. 15 No. 3, pp. 229-275.

Veroy, K. and Patera, A. (2005), "Certified real-time solution of the parametrized steady incompressible Navier-Stokes equations: rigorous reduced-basis a posteriori error bounds", Int. I. Numer. Meth. Fluids, Vol. 47 Nos 8-9, pp. 773-788.

\section{Appendix 1. PGD-based parametric solver}

In this appendix, we illustrate the PGD by considering the following parametric heat transfer equation:

$$
\frac{\partial u}{\partial t}-k \Delta u-f=0
$$

with homogeneous initial and boundary conditions. The enforcement of non-homogeneous initial and boundary conditions was deeply treated in Gonzalez et al. (2010) and Chinesta et al. (2010b).

Here $(\mathrm{x}, t, k) \in \Omega \times I \times \mathfrak{I}$, and the source term $f$ is assumed constant. The conductivity $k$ is viewed as a new coordinate defined in the interval $\mathfrak{\Im}$. Thus, instead of solving the thermal model for different discrete values of the conductivity parameter, we wish to solve at once a more general problem, the price to pay being an increase of the problem dimensionality. However, as the complexity of the PGD scales only linearly (and not exponentially) with the space dimension, 
consideration of the conductivity as a new coordinate still allows one to efficiently obtain an accurate solution.

The weak form related to Equation (A1) reads:

$$
\int_{\Omega \times I \times \mathfrak{J}} u^{*} \cdot\left(\frac{\partial u}{\partial t}-k \Delta u-f\right) d \mathbf{x} d t d k=0,
$$

for all test functions $u^{*}$ selected in an appropriate functional space.

The PGD solution is sought in the form:

$$
u(\mathbf{x}, t, k) \approx \sum_{i=1}^{N} X_{i}(\mathbf{x}) \cdot T_{i}(t) \cdot K_{i}(k) .
$$

At enrichment step $n$ of the PGD algorithm, the following approximation is already known:

$$
u^{n}(\mathbf{x}, t, k)=\sum_{i=1}^{n} X_{i}(\mathbf{x}) \cdot T_{i}(t) \cdot K_{i}(k) .
$$

We wish to compute the next functional product $X_{n+1}(\mathbf{x}) \cdot T_{n+1}(t) \cdot K_{n+1}(k)$, which we write as $R(\mathbf{x})$. $S(t) \cdot W(k)$ for notational simplicity.

Thus, the solution at enrichment step $n+1$ reads:

$$
u^{n+1}=u^{n}+R(\mathbf{x}) \cdot S(t) \cdot W(k) .
$$

We propose the simplest choice for the test functions $u^{*}$ used in Equation (A2):

$$
u^{*}=R^{*}(\mathbf{x}) \cdot S(t) \cdot W(k)+R(\mathbf{x}) \cdot S^{*}(t) \cdot W(k)+R(\mathbf{x}) \cdot S(t) \cdot W^{*}(k) .
$$

With the trial and test functions given by Equations (A5) and (A6), respectively, Equation (A2) is a non-linear problem that must be solved by means of a suitable iterative scheme. In our earlier papers (Ammar et al., 2006, 2007), we used Newton's method. Simpler linearization strategies can also be applied, however. The simplest one is an alternating direction, fixed point algorithm, which was found remarkably robust in the present context. Each iteration consists of three steps that are repeated until reaching convergence, that is, until reaching the fixed point. The first step assumes $S(t)$ and $W(k)$ known from the previous iteration and compute an update for $R(\mathrm{x})$ (in this case the test function reduces to $\left.R^{*}(\mathrm{x}) \cdot S(t) \cdot W(k)\right)$. From the just-updated $R(\mathrm{x})$ and the previously used $W(k)$, we can update $S(t)$ (with $u^{*}=R(\mathrm{x}) \cdot S^{*}(t) \cdot W(k)$ ). Finally, from the just computed $R(\mathrm{x})$ and $S(t)$, we update $W(k)$ (with $u^{*}=R(\mathrm{x}) \cdot S(t) \cdot W^{*}(k)$ ). This iterative procedure continues until reaching convergence. The converged functions $R(\mathrm{x}), S(t)$ and $W(k)$ yield the new functional product of the current enrichment step: $X_{n+1}(\mathrm{x})=R(\mathrm{x}), T_{n+1}(t)=S(t)$ and $K_{n+1}(k)=W(k)$. The explicit form of these operations is described as follows.

Computing $R(\mathrm{x})$ from $S(t)$ and $W(k)$.

We consider the weak form of Equation (A1):

$$
\int_{\Omega \times I \times \mathfrak{I}} u^{*}\left(\frac{\partial u}{\partial t}-k \Delta u-f\right) d \mathrm{x} d t d k=0 .
$$

here, the trial function is given by:

$$
u(\mathrm{x}, t, k)=\sum_{i=1}^{n} X_{i}(\mathrm{x}) \cdot T_{i}(t) \cdot K_{i}(k)+R(\mathrm{x}) \cdot S(t) \cdot W(k) .
$$

Since $\mathrm{S}$ and $\mathrm{W}$ are known from the previous iteration, the test function reads:

$$
u^{*}(\mathrm{x}, t, k)=R^{*}(\mathrm{x}) \cdot S(t) \cdot W(k) .
$$


Introducing (A8) and (A9) into (A7) yields:

$$
\begin{aligned}
& \int_{\Omega \times I \times \mathfrak{J}^{2}} R^{*} \cdot S \cdot W \cdot\left(R \cdot \frac{\partial S}{\partial t} \cdot W-k \cdot \Delta R \cdot S \cdot W\right) d \mathrm{x} d t d k \\
& \quad=-\int_{\Omega \times I \times \mathfrak{\Im}} R^{*} \cdot S \cdot W \cdot \mathscr{R}^{n} d \mathrm{x} d t d k,
\end{aligned}
$$

where $\mathcal{R}^{n}$ is the residual at enrichment step $n$ :

$$
\mathcal{R}^{n}=\sum_{i=1}^{n} X_{i} \cdot \frac{\partial T_{i}}{\partial t} \cdot K_{i}-\sum_{i=1}^{n} k \cdot \Delta X_{i} \cdot T_{i} \cdot K_{i}-f .
$$

since all functions involving time and conductivity have been determined, we can integrate Equation (A10) over $I \times \mathfrak{J}$. With the following notations:

$$
\left[\begin{array}{lll}
w_{1}=\int_{\mathfrak{J}} W^{2} d k & s_{1}=\int_{I} S^{2} d t & r_{1}=\int_{\Omega} R^{2} d \mathrm{x} \\
w_{2}=\int_{\mathfrak{\Im}} k W^{2} d k & s_{2}=\int_{I} S \cdot \frac{d S}{d t} d t & r_{2}=\int_{\Omega} R \cdot \Delta R d \mathrm{x} \\
w_{3}=\int_{\mathfrak{J}} W d k & s_{3}=\int_{I} S d t & r_{3}=\int_{\Omega} R d \mathrm{x} \\
w_{4}^{i}=\int_{\mathfrak{J}} W \cdot K_{i} d k & s_{4}^{i}=\int_{I} S \cdot \frac{d T_{i}}{d t} d t & r_{4}^{i}=\int_{\Omega} R \cdot \Delta X_{i} d \mathrm{x} \\
w_{5}^{i}=\int_{\mathfrak{S}} k W \cdot K_{i} d k & s_{5}^{i}=\int_{I} S \cdot T_{i} d t & r_{5}^{i}=\int_{\Omega} R \cdot X_{i} d \mathrm{x}
\end{array}\right],
$$

Equation (A10) reduces to:

$$
\begin{aligned}
& \int_{\Omega} R^{*} \cdot\left(w_{1} \cdot s_{2} \cdot R-w_{2} \cdot s_{1} \cdot \Delta R\right) d \mathrm{x} \\
& =-\int_{\Omega} R^{*} \cdot\left(\sum_{i=1}^{n} w_{4}^{i} \cdot s_{4}^{i} \cdot X_{i}-\sum_{i=1}^{n} w_{5}^{i} \cdot s_{5}^{i} \cdot \Delta X_{i}-w_{3} \cdot s_{3} \cdot f\right) d \mathrm{x} .
\end{aligned}
$$

Equation (A13) defines in weak form an elliptic steady state boundary value problem for the unknown function $R$ that can be solved by using any suitable discretization technique (finite elements, finite volumes, etc.). Another possibility consists in coming back to the strong form of Equation (A13):

$$
w_{1} \cdot s_{2} \cdot R-w_{2} \cdot s_{1} \cdot \Delta R=-\left(\sum_{i=1}^{n} w_{4}^{i} \cdot s_{4}^{i} \cdot X_{i}-\sum_{i=1}^{n} w_{5}^{i} \cdot s_{5}^{i} \cdot \Delta X_{i}-w_{3} \cdot s_{3} \cdot f\right),
$$

that can be solved by using any classical collocation technique (finite differences, SPH, etc....). Computing $S(t)$ from $R(\mathrm{x})$ and $W(k)$.

In the present case, the test function is written as:

$$
u^{*}(\mathrm{x}, t, k)=S^{*}(t) \cdot R(\mathrm{x}) \cdot W(k),
$$

and the weak form becomes:

$$
\begin{aligned}
& \int_{\Omega \times I \times \mathfrak{F}} S^{*} \cdot R \cdot W \cdot\left(R \cdot \frac{\partial S}{\partial t} \cdot W-k \cdot \Delta R \cdot S \cdot W\right) d \mathrm{x} d t d k \\
& \quad=-\int_{\Omega \times I \times \mathfrak{T}} S^{*} \cdot R \cdot W \cdot \mathcal{R}^{n} d \mathrm{x} d t d k .
\end{aligned}
$$

Integrating over $\Omega \times \mathfrak{I}$, one obtains:

$$
\begin{aligned}
\int_{I} S^{*} \cdot\left(w_{1} \cdot r_{1} \cdot \frac{d S}{d t}-w_{2} \cdot r_{2} \cdot S\right) d t \\
\quad=-\int_{I} S^{*} \cdot\left(\sum_{i=1}^{n} w_{4}^{i} \cdot r_{5}^{i} \cdot \frac{d T_{i}}{d t}-\sum_{i=1}^{n} w_{5}^{i} \cdot r_{4}^{i} \cdot T_{i}-w_{3} \cdot r_{3} \cdot f\right) d t .
\end{aligned}
$$


Equation (A17) represents the weak form of the ODE defining the time evolution of the field $S$ that can be solved by using any stabilized discretization technique (SU, Discontinuous Galerkin, etc.). The strong form of Equation (A17) reads:

$$
w_{1} \cdot r_{1} \cdot \frac{d S}{d t}-w_{2} \cdot r_{2} \cdot S=-\left(\sum_{i=1}^{n} w_{4}^{i} \cdot r_{5}^{i} \cdot \frac{d T_{i}}{d t}-\sum_{i=1}^{n} w_{5}^{i} \cdot r_{4}^{i} \cdot T_{i}-w_{3} \cdot r_{3} \cdot f\right) .
$$

Equation (A18) can be solved by using backward finite differences, or higher order Runge-Kutta schemes, among many other possibilities.

Computing $W(k)$ from $R(\mathrm{x})$ and $S(t)$.

The test function is now given by:

$$
u^{*}(\mathrm{x}, t, k)=W^{*}(k) \cdot R(\mathrm{x}) \cdot S(t),
$$

and the weak form becomes:

$$
\begin{aligned}
& \int_{\Omega \times I \times \mathfrak{I}} W^{*} \cdot R \cdot S \cdot\left(R \cdot \frac{\partial S}{\partial t} \cdot W-k \cdot \Delta R \cdot S \cdot W\right) d \mathrm{x} d t d k \\
& \quad=-\int_{\Omega \times I \times \mathfrak{T}} W^{*} \cdot R \cdot S \cdot \mathcal{R}^{n} d \mathrm{x} d t d k
\end{aligned}
$$

Integration over $\Omega \times I$ yields:

$$
\begin{aligned}
& \int_{\mathfrak{J}} W^{*} \cdot\left(r_{1} \cdot s_{2} \cdot W-r_{2} \cdot s_{1} \cdot k \cdot W\right) d k \\
& \quad=-\int_{\mathfrak{J}} W^{*} \cdot\left(\sum_{i=1}^{n} r_{5}^{i} \cdot s_{4}^{i} \cdot K_{i}-\sum_{i=1}^{n} r_{4}^{i} \cdot s_{5}^{i} \cdot k \cdot K_{i}-r_{3} \cdot s_{3} \cdot f\right) d k .
\end{aligned}
$$

Equation (A21) does not involve any differential operator. The corresponding strong form reads:

$$
\left(r_{1} \cdot s_{2}-r_{2} \cdot s_{1} \cdot k\right) \cdot W=-\left(\sum_{i=1}^{n}\left(r_{5}^{i} \cdot s_{4}^{i}-r_{4}^{i} \cdot s_{5}^{i} \cdot k\right) \cdot K_{i}-r_{3} \cdot s_{3} \cdot f\right) .
$$

This is an algebraic problem, which is hardly a surprise since the original Equation (A1) does not contain derivatives with respect to the parameter $k$. Introduction of the parameter $k$ as additional model coordinate does not increase the cost of a particular enrichment step. It does, however, necessitate more enrichment steps, i.e. more terms (higher $N$ ) in the decomposition (A3).

We have seen that at each enrichment step the construction of the new functional product in Equation (A3) requires non-linear iterations. If $m_{i}$ denotes the number of iterations needed at enrichment step $i$, the total number of iterations involved in the construction of the PGD approximation is $m=\sum_{i=1}^{i=N} m_{i}$. In the above example, the entire procedure thus involves the solution of $m 3 \mathrm{D}$ problems for the functions $X_{i}(\mathbf{x}), m 1 \mathrm{D}$ problems for the functions $T_{i}(t)$ and $m$ algebraic systems for the functions $K_{i}(k)$. In general, $m$ rarely exceeds ten. The number $N$ of functional products needed to approximate the solution with enough accuracy depends on the solution regularity. All numerical experiments carried to date reveal that $N$ ranges between a few tens and 100. Thus, we can conclude that the complexity of the PGD procedure to compute the approximation (A3) is of some tens of 3D steady state problems (the cost related to the $1 \mathrm{D}$ and algebraic problems being negligible with respect to the $3 \mathrm{D}$ problems). In a classical approach, one must solve for each particular value of the parameter $k$ a 3D problem at each time step. In usual applications, this often implies the computation of several millions of 3D solutions. Clearly, the CPU time savings by applying the PGD can be of several orders of magnitude.

\section{Appendix 2. Dimensionless Rayleigh-Benard problem}

Let's be the mass, momentum and energy balances: 


$$
\left\{\begin{array}{l}
\nabla \cdot \mathbf{v}=0 \\
\rho\left(\frac{\partial \mathbf{v}}{\partial t}+\mathbf{v} \cdot \nabla \mathbf{v}\right)=-\nabla p+\nabla \cdot \tau+\rho \cdot g \cdot \beta \cdot\left(T-T_{r}\right) \mathbf{j} \\
\frac{\partial T}{\partial t}+\mathbf{v} \cdot \nabla T=\alpha \cdot \nabla^{2} T
\end{array}\right.
$$

where $\mathbf{v}$ is the velocity field, $p$ is the pressure, $T$ the temperature, $\mathbf{D}$ the strain rate tensor (symmetric component of the velocity gradient), $\alpha$ the thermal diffusivity $\left(\alpha=(k) /\left(\rho \cdot C_{p}\right), k\right.$ being the thermal conductivity - assumed isotropic, $\rho$ the density and $C_{p}$ the specific heat), $\tau$ the deviatoric part of the Cauchy's stress tensor, $\mathrm{g}$ the gravity's acceleration, $\beta$ the expansion coefficient, $T_{r}$ a reference temperature and $\mathbf{j}$ the unit vector defining the $y$-direction along which the gravity applies.

The constitutive equation for a Newtonian fluid reads:

$$
\tau=2 \cdot \eta \cdot \mathbf{D}
$$

The dimensionless form of these equations is performed by considering the following relations, in which the star superscript refers to the dimensionless variables:

$$
\left\{\begin{array}{l}
\mathbf{x}=\mathbf{x}^{*} \cdot H \\
t=t^{*} \cdot \frac{H}{(g \cdot \beta H \cdot \Delta T)^{\frac{1}{2}}} \\
\mathbf{v}=\mathbf{v}^{*} \cdot(g \cdot \beta H \cdot \Delta T)^{\frac{1}{2}} \\
p=p^{*} \cdot(g \cdot \beta H \cdot \Delta T) \cdot \rho \\
T=\theta \cdot \Delta T+T_{r}
\end{array}\right.
$$

where $H$ is the length of the square cavity in which the flow takes place, $\Delta T$ the temperature difference between the upper and the bottom boundaries having temperatures $T_{c}$ and $T_{H}$ $\left(T_{H}>T_{C}\right)$, respectively. In this work we considered $T_{r}=\left(T_{C}+T_{H}\right) / 2$.

By introducing relations (A25) into the balance Equations (A23) and making use of the dimensionless Prandtl $\left(P_{r}\right)$ and Rayleigh $\left(R_{a}\right)$ numbers, the first defined from the ratio of momentum diffusivity to thermal diffusivity, and the second one related with buoyancy-driven flows:

$$
\left\{\begin{array}{l}
P_{r}=\frac{2 \cdot \eta \cdot C_{p}}{k} \\
R_{a}=\frac{g \cdot \beta \cdot H^{3} \cdot \Delta T \cdot \rho}{2 \cdot \eta \cdot \alpha}
\end{array}\right.
$$

by omitting star superscripts the dimensionless Rayleigh-Benard model finally writes:

$$
\left\{\begin{array}{l}
\nabla \cdot \mathbf{v}=0 \\
\frac{\partial \mathbf{v}}{\partial t}+\mathbf{v} \cdot \nabla \mathbf{v}=-\nabla p+\left(\frac{P_{r}}{R_{a}}\right)^{\frac{1}{2}} \cdot \nabla \cdot \mathbf{D}+\theta \mathbf{j} \\
\frac{\partial \theta}{\partial t}+\mathbf{v} \cdot \nabla \theta=\left(P_{r} \cdot R_{a}\right)^{-\frac{1}{2}} \cdot \nabla^{2} \theta
\end{array}\right.
$$

